\title{
Revision der Gattung Nanna BIRKET-SMith, 1965 (Lepidoptera, Arctiidae, Lithosiinae)
}

\author{
Mit 9 Figurentafeln
}

LARS KÜHNE ${ }^{1}$

${ }^{1}$ Stephensonstraße 29, 14482 Potsdam-Babelsberg, Deutschland

Published on 2014-07-31

\section{Zusammenfassung}

Die afrotropische Gattung Nanna Birket-Smith, 1965 wird revidiert. Sieben Arten und zwei Unterarten werden neu beschrieben: broetheri sp. nov., hoppei sp. nov., distyi sp. nov., eningae albifrons ssp. nov., eningae subflava ssp. nov., tanzaniae sp. nov., luteolata sp. nov. griseoides sp. nov. und falcata sp. nov. Der Status von pia (STRAND, 1912) wird revidiert (stat. rev.) und ein Lectotypus designiert und das Taxon magna Birket-Smith, 1965 als Synonym (syn. nov.) von pia STRAND gewertet.

\section{Summary}

The Afrotropical genus Nanna Birket-Smith, 1965 is revised. Seven new species and two new subspecies are described: broetheri sp. nov., hoppei sp. nov., distyi sp. nov., eningae albifrons ssp. nov., eningae subflava ssp. nov., tanzaniae sp. nov., luteolata sp. nov. griseoides sp. nov. and falcata sp. nov. The status of pia (STRAND, 1912) is revised (stat. rev.) and a lectotype is signed. The taxon magna BIRKeT-SMith, 1965 is a synonym (syn. nov.) of pia STRAND.

\section{Key words}

Lepidoptera, Arctiidae, Lithosiinae, genus Nanna, revision, taxonomy, Africa

\section{Einleitung}

Seit der Abhandlung der Lithosiinae im Seitz'schen Werk (1926) wurde im letzten Jahrhundert nur wenig Wissen für den afrikanischen Kontinent hinzugefügt. Derzeit sind etwa 500 Arten bekannt. Neben den taxonomischen Arbeiten von Kiriakoff (1954a, 1954b, 1958, 1963) stellt die Bearbeitung der westafrikanischen "Eilemic group" von Birket-Smith (1965) die umfangreichste dar. In dieser Arbeit errichtete er auch die Gattung Nanna.

Neuere Forschungen (Roesler 1990, Durante \& Panzera 2001a, 2001b, 2002a, 2002b, 2002c, Durante
2007, 2009, 2012, KüHNE 2005a, 2007, 2008) haben gezeigt, dass die Taxonomie der afrikanischen Flechtenbären bei Weitem noch nicht aufgeklärt ist. Sie legen nahe, dass schätzungsweise ein Drittel der afrikanischen Arten noch gar nicht beschrieben ist. Ein vorläufiges Endergebnis ist zu erwarten, wenn die fertiggestellte Revision der Gattung Cyana von TIMm KARIScH (Dessau, Germany) erscheint und die aller Lithosiini durch MARTIN KRÜGER (Pretoria, RSA) fertig gestellt ist. Zum letztgenannten Vorhaben ist die hier vorgestellte Arbeit ein verabredeter Beitrag. 
Unzweifelhaft ist, dass die Lithosiinae innerhalb der Familiengruppe Arctiidae einzuordnen ist. Der Rang wird in letzter Zeit verschieden gehandhabt. Der Betrachtung als Unterfamilie bei JACOBSEN \& WeLler (2002), Bendib \& Minet (1999) und Holloway (2001) liegen fundierte Untersuchungen zu Grunde. Eine zuletzt geäußerte Ansicht, die Gruppe im Rang einer Untertribus zu führen (LAFONTAINE \& FibIGER, 2006), berücksichtigt nicht, dass phylogenetische Hypothesen sich nicht ohne Weiteres mit den Rangstufen der Klassifikation abbilden lassen, sondern vielmehr dadurch nur die Auffindbarkeit von Informationen erschwert wird. BENDIB \& MINET (1999) unterteilen die Lithosiinae in sieben Gattungsgruppen. Die Gattungsgruppe der Lithosiini - manchmal auch als Eilemini bezeichnet - umfasst in Afrika derzeit etwa 280 Taxa.

Zur Gattung Nanna gehören mit die größten Flechtenbärenarten. Einige erreichen Spannweiten von fast sieben Zentimeter und erinnern stark an die Männchen der paläarktischen Lithosia quadra (LinnaEus, 1758). Andere Arten haben „normale“ Lithosiengröße von etwa vier Zentimeter. Sehr charakteristisch sind zwei dunkle Punkte im Vorderflügel bei beiden Geschlechtern. Die Grundfarbe reicht von grau bis gelb.

Ursprünglich war im Rahmen der Revision auch geplant, eine phylogenetische Hypothese für die untersuchte Gattung vorzustellen, weil die Arten hauptsächlich den tropischen Regenwald bzw. den Waldrand bewohnen und phylogenetisch interessante Verbreitungsmuster haben. Sie sind offensichtlich das Ergebnis jüngerer Radiationen im Zusammenhang mit der Kontraktion und Ausdehnung des Regenwaldes. Somit hätte dies einen weiteren Baustein für Speziationsmuster liefern können. Leider ergab die Analyse der verfügbaren Merkmale (Flügelzeichnung und Bau der Genitalarmatur beider Geschlechter), dass zahlreiche Parallelismen vorliegen. Die Ergebnisse waren derart konträr, dass keine vertretbare phylogenetische Hypothese erarbeitet werden konnte. Allerdings sind Gruppierungen nahe verwandter Arten möglich und die Reihenfolge der nachstehenden Abhandlung folgt diesen Kenntnissen.

\section{Methodik}

Für die Untersuchung wurde das komplette Typenmaterial sowie umfangreiches Ausleihmaterial aus verschiedenen Sammlungen untersucht. Es wurden Dauerpräparate der Genitalarmaturen angefertigt (Einbettung in Euparal). Die Termini im Text entsprechen denen von KüHne (2005b). Die Abbildungen wurden mittels Photoshop erstellt.

$\begin{array}{ll}\text { Verwendete Abkürzungen: } \\ \text { GP } \quad \text { Genitalpräparat } \\ \text { Vdfl. } & \text { Vorderflügel } \\ \text { Hfl. } & \text { Hinterflügel } \\ \text { HT } & \text { Holotypus }\end{array}$

\section{Verwendete Abkürzungen:}

GP Genitalpräparat

Vdfl. Vorderflüge

HT Holotypus

$\begin{array}{ll}\text { PT } & \text { Paratypus/Paratypen } \\ \text { ZSM } & \text { Zoologische Staatssammlung, München } \\ \text { MRAC } & \text { Musee Royal de l'Afrique Centrale, Tervuren } \\ \text { MfN } & \text { Museum für Naturkunde, Berlin } \\ \text { NKMD } & \text { Naturkundemuseum Dessau } \\ \text { BMNH } & \text { British Museum of Natural History, London } \\ \text { NHRS } & \text { Naturhistoriska Riksmuseet, Stockholm } \\ \text { SMNS } & \text { Staatliches Museum für Naturkunde, Stuttgart } \\ \text { LKP } & \text { coll L. Kühne, Potsdam } \\ \text { CI } & \text { Elfenbeinküste } \\ \text { DRC } & \text { Demokratische Republik Kongo } \\ \text { UG } & \text { Uganda } \\ \text { AO } & \text { Angola } \\ \text { KE } & \text { Kenia } \\ \text { CM } & \text { Kamerun }\end{array}$

\section{Taxonomische Ergebnisse}

Nanna Birket-Smith, 1965: 35

Gattungstypus: Ilema diplisticta BETHUNE-BAKER, 1911

Die Gattung umfasste bisher folgende Taxa (in alphabetischer Reihenfolge):

ceratopygia BiRKet SMITH, 1965

collinsii KüHNE, 2007

colonoides (KIRIAKOFF, 1963)

diplisticta (Bethune-BAKer, 1911)

eningae (РцÖTZ, 1880)

griseata KüHNE, 2007

kamerunica KüHNE, 2007

loloana (STRAND, 1912)

magna BiRKeT-SMITH, 1965

melanosticta (BETHUNE-BAKER, 1911)

montana BIRKET-SMITH, 1965

naumanni KüHNE 2005

pia STRAND, 1912 (var.)

\section{Allgemeine Gattungsbeschreibung}

(Diese Angaben werden im Text bei den einzelnen Arten nicht mehr wiederholt.)

Habitus: Die Spannweite der Arten reicht von 25-65 mm, wobei die Weibchen oft markant größer sind als die Männchen. Damit umfasst die Gattung die größten Vertreter der Familiengruppe. Typisch für die Gattung sind zwei schwarz(braune) Punkte im Vdfl. und der im basalen Drittel dunkel gefärbte Costalrand. Ein Punkt liegt direkt vor dem vorderen Flügelrand, geht aber farblich nicht in diesen über. Dieses Merkmal ist lediglich bei einigen Exemplaren von $N$. ceratopygia verwischt. Der zweite Punkt befindet sich nahe der Analader des Vdfl. Die Punktanordnung bei den Gattungen, die gleichfalls 
zwei Punkte im Vdfl. besitzen, z. B. Ilema, Cragia, Zobida, ist anders gestaltet.

Bei der Farbausprägung gibt es drei Hauptformen: grau, hell ockergelb und gelb. Überwiegend sind die Hfl. heller als die Vdfl., bei einigen Arten sogar viel heller als die Vdfl. Kopf, Thorax und Abdomenende sind gelblich beschuppt, die kraniale Abdomenhälfte ocker bis grau. Die Beine sind bei etlichen Arten distal dunkel bis schwarz beschuppt.

đ Genitalien: Uncus normal gebaut, am Ende mit kleiner Spitze. Tegumen als breites Band, meist lateral verschmälert, Vinculum als schmale, ventral stark sklerotisierte Spange. Die Valven zweigeteilt, der dorsale Teil schwächer sklerotisiert, dadurch oft häutig erscheinend, der ventrale Teil besonders distal stärker sklerotisiert, oft zugespitzt und gebogen, hier bei einigen Arten kräftig, kurz behaart. Eine markante Bildung an der Costabasis der inneren Valve ist bei vielen Arten ein stark sklerotisierter Fortsatz, der nach dorsocaudal gerichtet ist (Proc. basalia plicae sensu Birket-SMith 1965). Er fehlt nur bei den Arten um eningae. Die Juxta ist im Prinzip eine dreieckige Platte mit abgerundeten Ecken, deren ventrale Hypotenuse mittig ausgebuchtet ist. Der Aedoeagus ist kurz und gedrungen, dorsal besitzt er einen am Ende abgerundeten Fortsatz. Die Vesica ist einfach gebaut und partiell mit kleinen Dornenfeldern ausgestattet.

† Genitalien: Die Legeröhre ist kurz, beide Apophysenpaare sind zart gebaut und kaum länger als das jeweilige Segment. Das 8. Segment ist ventral nur schwach sklerotisiert und hier meist häutig. Das Ostium befindet sich in der Intersegmentalhaut kranial vom 8. Segment und ist häutig. Gelegentlich tritt kaudal vom Ostium eine prominente, sekundäre Sklerotisierung der Intersegmentalhaut auf, die plattenförmig ventral im 8. Segment liegt. Der Ductus bursae ist kurz, meist mit einem Antrum, das Corpus bursae ist einfach oval und innen überwiegend mit kleinen Dornen besetzt. Oft ist ein Signum vorhanden.

\section{Nanna diplisticta (BETHUNE-BAKER, 1911) Tafeln 1, 3}

\section{Ilema diplisticta BETHUNE-BAKER, 1911: 535}

Material: HT, o", "Gunnal, / Portugese W. Africa, /June 1910”, Präp: 757 (Arc.), [BMNH]; CI: 1 ㅇ, Agboville, 12.-18.04.1969, C. Herbulot, Präp. 792 [ZSM].

Die Art ist im männlichen Geschlecht bisher nur vom Typus bekannt. BIRкET-SMITH (1965) bildet fälschlicherweise ein GP von colonoides (KIRIAKOFF, 1963) ab. Mit Unsicherheit behaftet bleibt die Lokalisierung des Typenfundortes (Gunnal), weil der Sammler William John Ansorge (1850-1913) oft und innerhalb kurzer Zeit seine Aufenthaltsorte zwischen Angola, Gabun und Guinea-Bissau wechselte. Dies ergab eine Auswertung der Lokalitäten, an denen er anderes Tiermaterial
(Fische, Vögel, andere Lepidopteren) gesammelt hat (vgl. Boulenger, 1905, 1906, 1910, 1911a, 1911b; Kirby, 1896; Dean et al. 2003, 2009). Derartige Ortswechsel waren zur damaligen Zeit mit den Postdampfern fast unproblematisch und zügig möglich. Seine persönlichen Aufzeichnungen befinden sich in London, wurden aber im Rahmen dieser Arbeit nicht eingesehen.

In der Ornithologie wird "Gunnal“ (jetzt Gunal) einheitlich als „besiedelter Platz" in Guinea-Bissau verortet (M. Honey, S. Frahnert, schriftliche Mitteilung). Aufgrund der damals üblichen Fortbewegung Forschungsreisender in Schiffen, Lastkähnen und Booten auf den Flusssystemen entlang der Handelsrouten bzw. -niederlassungen, verorte ich den Typusfundort leicht abweichend hier mit „Rio de Gunal“ $\left(12.25^{\circ} \mathrm{N}\right.$, $\left.15,8333^{\circ} \mathrm{W}\right)$, der in den Rio Cacheu unterhalb Sao Vincente mündet.

Habitus: Spannweite $28 \mathrm{~mm}$, Vdfl. etwas rundlich, graubraun. Die Punkte stehen fast in einer parallelen Linie zum Körper, der vordere ist schwächer ausgebildet. Der Hfl. weißlich, zum Rand hin zunehmend dunkler beschuppt. Die Variabilität der Art ist unbekannt.

o Genitalien: Uncus etwa so lang wie Tegumen, der dorsale Valventeil gerade, am Ende nicht erweitert und dorsal etwas abgerundet, ventral leicht spitz zulaufend. Der ventrale Valventeil stark sklerotisiert und gleichmäßig kurz aufgebogen, hier bedornt. Der innere Fortsatz gerade, dünn, spitz, zerstreut bedornt, etwa halb so lang wie die Valve.

† Genitalien: Mit großer Sicherheit handelt es sich bei dem abgebildeten Tier um das bisher unbekannte Weibchen dieser Art. Die Zuordnung erfolgte aufgrund des Fundortes und der Ähnlichkeit in den Merkmalskombinationen zu den nahe stehenden Arten.

Differentialdiagnose: Im Habitus von den ähnlichen Arten nicht sicher zu trennen. Innere Fortsätze der Valve im Vergleich zur ähnlichen loloana STRAND, 1912 doppelt so dick und Uncus länger.

\section{Nanna loloana (STRAND, 1912)}

Tafeln 1, 3

\section{Phryganopsis loloana STRAND, 1912: 172}

Material: HT, ㅇ, „S. Kamerun/Lolodorf/L. Conradt S.“, [ohne Hinterleib], [MfN]; CM: $1 \sigma^{\star}$, Adamaua region, [MfN]; 1 o , Lolodorf, 1894-1895, L. Conradt, [BMNH]. Die Exemplare am MfN in Berlin sind stark zerstört. Vermutlich wurde eine Rücksendung an das Museum aus dem BMNH auf dem Postweg so stark beschädigt, dass nur noch die Leiber auf den Nadeln existieren. Die Präparate sind unversehrt. Das betrifft auch andere Typen der Lithosiinae. Zum Glück gibt es am BMNH noch schwarz/ weiß-Fotos von den unzerstörten Typen, so dass in den 
meisten Fällen eine sichere Identifikation der Taxa gewährleistet ist.

Entgegen der Etikettierung mit roten Typenschildern und den Angaben bei Birket-Smith (1965) hat Strand (1912: 172, 173) nur das Weibchen von Lolodorf als Typus ausgewiesen. Das „nachträglich aufgefundene Männchen von Adamaua" hat Strand (ebenda) lediglich als fast identisch bezeichnet. Der weibliche (Holo)Typus ist bis auf das Geschlecht aufgrund der Zerstörung nicht mehr verifizierbar. Im Fall von loloana gibt es am BMNH noch einen Topotypus, designiert von Fletcher. Dessen Genitalarmatur wurde abgepinselt und untersucht. Sie stimmt mit dem des Männchens aus Adamaua überein. Die Interpretation von loloana erfolgt deshalb auf der Basis der Genitalarmatur des von Strand selbst zugeordneten Männchens in Kombination mit dem Topotypus von Fletcher. Die Genitalabbildung des Männchens bei Birket-Smith (1965: 40) ist korrekt.

Habitus: Spannweite Männchen $30 \mathrm{~mm}$, Weibchen $33 \mathrm{~mm}$, Vdfl. graubraun, Hfl. hell, am Vorderand breit dunkler bestäubt. Das Abdomen fast vollständig gelb beschuppt.

o Genitalien: Uncus halb so lang wie Tegumen, schmal, gerade mit kurzer Spitze, Tegumen gleichmäßig breit. Valve nur bis etwa $1 / 3$ geteilt, der ventrale Fortsatz stark sklerotisiert und aufwärts gebogen, mit zahlreichen kleinen Dornen. Der innere Fortsatz der Costabasis dünn, stark zugespitzt, ganz leicht gebogen, knapp 2/3 so lang wie die Valve. Aedoeagus kurz, schwach gebogen, ventral mit einem Dornenfeld.

\section{ㅇ Genitalien: unbekannt}

Differentialdiagnose: Nur mittels GP in dieser Artengruppe sicher zu identifizieren, Uncus viel kürzer als bei diplisticta, innerer Fortsatz der Valvenbasis dünner und etwas länger.

\section{Nanna ceratopygia BIRKET-SMITH, 1965}

Tafeln 1, 3

Nanna ceratopygia Birket-Smith, 1965: 40

Material: HT, ơ, “Bitje, Ja River/Cameroons/1915., Präp. 887, [BMNH]; CM: 1 o, Bitje, Ja River, Oct-Nov.

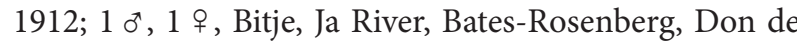
M. Torley [MRAC, LKP].

Habitus: Spannweite Männchen 35-38 mm, Weibchen 40-45 mm, Grundfarbe der Vdfl. graubraun, Hfl. gelblich. Costalrand nur bei den Männchen ganz dünn verdunkelt, Costalpunkt kräftig dunkelbraun, fast schwarz, mit der Costa leicht zerfließend, Analpunkt etwas schwächer, gelegentlich ganz klein. Kopf schwach gelblich gefärbt, Thorax in der Farbe der Vdfl.,
Abdomen etwas heller grau und nur am Ende schwach gelblich. Beine graubraun.

o Genitalien: Uncus mittellang, Tegumen fast gleichbreit, Valve etwa zu 1/4 geteilt, dorsaler Valventeil zugespitzt und mehr sklerotisiert, ähnlich wie bei den folgenden Arten. Der ventrale Valventeil in der Mitte stark verbreitert, dann plötzlich stark verjüngt. Dadurch wirkt der rechtwinklig gebogene, stark sklerotisierte und dicht behaarte Fortsatz wie abgesetzt. Der innere Valvenfortsatz in der Grundform ähnlich wie bei diplisticta und loloana, aber an der Basis stärker und hier seitlich mit einem doppelten so langen, dünnen Fortsatz, der den inneren Fortsatz gegabelt erscheinen lässt. Aedoeagus kurz, der distale sklerotisierte Fortsatz ganz kurz, distal quasi nur als kopfartige Auswölbung ausgebildet, er setzt sich körperwärts als Leiste mit kurzen Zähnen fort.

† Genitalien: 8. Segment schmal, ventral häutig, hier mit einer breiten, fast rechteckigen Platte. Das breite Ostium schließt sich direkt an und wird seitlich von den leicht umgeschlagenen Rändern des 7. Segments eingefasst. Ductus bursae sehr kurz, Corpus bursae oval, innen vollständig dicht bedornt, mit einem kräftigen, rundlichen Signum in der Mitte.

Differentialdiagnose: Habituell durch die Größe und die bräunliche Färbung sicher zu bestimmen.

\section{Nanna colonoides (KIRIAKOFF, 1963)}

Tafeln 1, 4

Lithosia colonoides KIRIAKOFF, 1963: 101

= diplisticta sensu Birket Smith (1965) (die GP-Abbildung)

Material: HT, ơ , riv. Kombo, affl. Ruanoli, 1550 m, 19.vii.1954, Präp. 26, [MRAC]; CG: 2 o $^{\star}$ Odzala Nat. Park, 29.01.-03.03.1997, Siniaev \& Murzin, [ZSM]; DRC: $2 \sigma^{\star}$, Forèt de Djugu, 11.v.1953, J. Hecq, [MRAC]; DRC: 5 ơ, Paulis (Uele), 5.ii.1960, M. Fontaine, [MRAC]; CI: $1 \sigma^{\star}$, Lamto, 24.8.74, de Toulgeot, [ZSM]; $1 \sigma^{\star}$, Forêt classée Bossematié, 10/1993, [MRAC]; $10^{\star}$, ix.2004, E. Vingerhoedt, UG: $1 o^{\star}$, Fort Portal, 26.-28.12.1991,

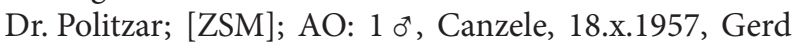
Heinrich, [LKP]; DRC: 1 \%, Dimbelenge, 20.i.1958, Dr. M. Fontaine, [LKP].

Sowohl in Größe, Flügelfärbung und bei den inneren Fortsätzen am männlichen GP ist dieses Taxon derart variabel, dass die These, ob mehrere Arten vorliegen, zu prüfen war. Die typologische Gruppierung des vorliegenden Materials (15 Tiere) anhand ausgewählter Merkmale war in der Gesamtbetrachtung nie widerspruchsfrei. Auch ergaben sich bei der geografischen Interpretation des jeweils so sortierten Materials keine vertretbaren Verbreitungsmuster. Gestützt wird die hier vertretene Ein-Art-Hypothese mit einer ähnlichen Varianz der inneren Fortsätze am männlichen GP bei N. broetheri sp. nov. 
Man muss trotzdem einräumen, dass der Verdacht einer Superspecies nicht ausgeschlossen werden kann, aber derzeit nicht verifizierbar ist.

Habitus: Variable Art, Spannweite Männchen 26-37 mm, Weibchen 30-35 mm, Vdfl. grau, Hfl. weißlich, gelegentlich mit grauen Rand bzw. grau überstäubt, Punkte im Vdfl. meist parallel zum Körper, selten aber auch der Punkt an der Analis nach innen versetzt. Gelbfärbung des Abdomens variabel, Fühler gelblich.

$0^{\star}$ Genitalien: Uncus mittelang, Valve etwa bis zur Hälfte geteilt, ventraler Teil breit, spitz auslaufend, hier leicht aufwärts gebogen und stärker sklerotisiert. Die inneren Fortsätze der Valve stark sklerotisiert, fast so lang wie die Valve, kräftig, kurz behaart, besonders ventral, im Ansatz leicht gebogen, je nach Lage im GP manchmal auch gerade erscheinend, am Ende mit kleiner dünner Spitze. Aedoeagus kurz, der distale Fortsatz lang und schmal, an der ventralen Ausstülpung der Vesica mit 3-6 kurzen Dornen.

i Genitalien: Ostium tief in das 7. Segment eingesenkt und dessen Ränder schräg an der Einsenkung umgeschlagen. Diese Struktur erscheint im GP als breite, randlich geriffelte, stark sklerotisierte Einfassung, die nach kaudal auseinander läuft. Der Ductus bursae geht ansatzlos in das Corpus bursae über. Sklerotisierung im Ductus (Antrum) lang gezogen kaudal auf die Hälfte verjüngt. Corpus bursae innen stark bedornt, mit Signum, dessen Umgebung unbedornt ist.

Differentialdiagnose: Innerer Fortsatz der Valven im Unterschied $\mathrm{zu}$ den äußerlich ähnlichen diplisticta und loloana deutlich kräftiger gebaut, im Unterschied $\mathrm{zu}$ hoppei sp. nov. am Ende aber gerade auslaufend, bei den Weibchen das Antrum im Ductus bursae größer und proximal nicht auf die Hälfte verschmälert.

\section{Nanna broetheri sp. nov.}

Tafeln 1, 4

HT: $\sigma^{\star}$, „Rwanda / Nyungwe /8-3-75 / B. Turlin / 2000“ [ZSM].

PT: 4 ơ, 1 ㅇ , ebenda (x.[19]74-iv.[19]77), Präp. 803, 618, 620 [ZSM, LKP].

Beschreibung, Habitus: Die auffälligste Art in dieser Gruppe aufgrund ihrer kontrastreichen Zeichnung. Spannweite Männchen 28-36 mm, Weibchen 39-41 mm, hell gelbweiß, Hfl. ein wenig heller, die orangegelbe Thoraxfärbung zieht leicht in den Vdfl.-ansatz. Der Costalrand als dicker schwarzer Strich, fast den Costalpunkt erreichend, gelegentlich mit diesem verschmolzen. Analpunkt nach innen versetzt, gleichfalls sehr kräftig und schwarz. Tibien verdunkelt. $\sigma^{*}$ Genitalien: Kaum von colonoides verschieden, Uncus lang und halbrund gebogen, Valventeilung nur etwas bis $1 / 3$, ventraler Teil breit, spitz auslaufend, aufwärts gebogen und stärker sklerotisiert. Die inneren Fortsätze der Valve stark sklerotisiert, fast so lang wie die Valve, kräftig kurz behaart, besonders ventral, im Ansatz leicht gebogen, am Ende gerade und mit kleiner dünner Spitze. Juxta breit und den Aedoeagus in seiner natürlichen Lage weit umschließend, Aedoeagus kurz, der distale Fortsatz lang und schmal.

i Genitalien: Ostium tief in das 7. Segment eingesenkt und dessen Ränder schräg an der Einsenkung umgeschlagen, schmaler als bei colonoides und hoppei sp. nov. und kaudal leicht eckig vorgezogen, auch die Öffnung insgesamt breiter, Rand fast gerade, nur schwach geriffelt. Ductus bursae breit, ansatzlos in das Corpus bursae übergehend. Im Ductus bursae befindet sich ein Antrum, welches sich proximal auf fast $1 / 3$ verjüngt. Corpus bursae innen stark bedornt mit einem Signum, dessen Umgebung unbedornt ist.

Differentialdiagnose: Habituell sofort kenntlich durch die kontrastreiche Zeichnung, im Genital nur schwer von colonoides zu unterscheiden.

Derivatio nominis: Gewidmet und benannt nach Dr. Helmut Bröther (Potsdam). Er arbeitete bis zu seiner Pensionierung 2011 im Pflanzenschutzamt des Landes Brandenburg.

\section{Nanna naumanni KüHNE, 2005}

Tafeln 1, 4

Nanna naumanni KüHNE, 2005a: 490

Material: HT, ơ, Kenia, Kakamega Forest N.R., 27.iii.2002, leg. L. Kühne [LKP], 5 PT ebenda; KE: $1 \sigma^{\star}$, Kakamega Forest, 9.1.73, Politzar, [ZSM]; 1 ㅇ , Kakamega Forest, 13.x.2001, Präp. 562, L. Kühne, [LKP].

Die vorliegende Untersuchung ergab, dass sich unter naumanni zwei Arten verbergen. Demzufolge wird ein männlicher Paratypus von naumanni zu hoppei sp. nov. gestellt. Die beiden weiblichen Paratypen von naumanni werden ebenfalls zu hoppei sp. nov. gestellt, sie werden aber nicht in die Typenserie aufgenommen (siehe dort). Die Zuordnung der wenigen vorhandenen Weibchen zu den Taxa naumanni bzw. hoppei sp. nov. bleibt mit großen Unsicherheiten behaftet, weil beide Arten am gleichen Standort (z. B. Kakamega) vorkommen. Anhaltspunkte für die hier vorgenommene Zuordnung ist die Größe der Weibchen, die etwas spitzeren Vdfl. und die Hfl.Färbung. Insofern ist die Überstellung der weiblichen Paratypen von naumanni zu hoppei sp. nov. derzeit als die beste Diagnose - aber nicht als abschließend - zu betrachten. Diese Frage kann nur durch Zuchten oder weiteres Material von anderen Standorten geklärt werden. 
Habitus: Äußerlich ähnlich der diplisticta und colonoides, etwas kleiner, Spannweite 29-33 mm, Weibchen ein wenig größer. Vdfl. grau, Costa an der Basis schwarz und etwas distal von der Mitte mit schwarzen Costalpunkt, Analpunkt ganz leicht nach innen versetzt. Fühler, Beine, Palpen, Kopf, Thorax und Abdomen gelb, letzteres in der Mitte etwas dunkler grau.

○ Genitalien: Uncus hoch gebaut, seitlich stark abgeflacht, aufgewölbt. Tegumen breit, Valve am Ende zweigeteilt, ventral zugespitzt und stärker sklerotisiert. Innerer Fortsatz lang hakenförmig, kräftig sklerotisiert, das Tegumen überragend, ventral mit einer breiten Reihe kurzer Haare. Aedoeagus kurz.

† Genitalien (möglicherweise hoppei sp. nov.): Apophysen kurz, 8. Segment ventral häutig, Ostium in der Intersegmentalhaut, 7. Segment stark ausgerandet (im GP leider umgeschlagen), die vorgewölbten Ränder fast glatt, dazwischen mit einer flächigen Sklerotisierung. Ductus burase breit, das bedornte, kugelförmige Corpus bursae etwa gleich lang. Antrum den Ductus bursae in fast ganzer Breite ausfüllend und noch in das Corpus bursae ziehend, hier plötzlich verjüngt und nun dachrinnenförmig.

Differentialdiagnose: Hfl. meist fast weiß, Vdfl. insgesamt etwas spitzer als bei den vorigen Arten, dadurch schlanker wirkend. Der innerer Fortsatz am männlichen Genital ermöglicht ohne Präparation (Genitalien abpinseln) eine sichere Diagnose. Beim weiblichen Genital ist das breitere und kürzere Antrum gegenüber hoppei sp. nov. markant.

\section{Nanna hoppei sp. nov.}

Tafeln 1, 5

Material: HT: $\sigma^{\star}$ „coll. mus. congo / Sankuru: KatakoKombe / 9-X-1952 / Dr M. Fontaine“, Präp. 567, [MRAC] PT: 1 ๙ $^{\star}$, ebenda, 18-xii-1951; 1 o $^{\star}$, Kenya, Kakamega Forest, 13.x.2001, U. Dall'Asta, Präp. 605; $10^{\star}$ ebenda, 2.1.73, Plitzar, Präp. 607; 1 đ PT N. naumanni Kühne 2005, Kakamega Forest, Präp. 608, 2 o*, Congo, Odzala [OOzala sic.] Nat. Park, 1997. leg. Siniaev \& Murzin Präp. 609; [MRAC, LKP].

Weiteres Material, aus der Typenserie ausgeschlossen: 2 PT ㅇ N. naumanni Kühne 2005, Kakamega Forest, Präp. 606, 667; 1 ㅇ, Congo, Odzala [OOzala sic.] Nat. Park, 1997. leg. Siniaev \& Murzin Präp. 622.

Beschreibung, Habitus: Etwas kleiner als naumanni, Spannweite $25-28 \mathrm{~mm}$. Grundfarbe der Vdfl. in der Tendenz dunkler, mehr graubraun, oft ist der schwarze Costalrand an der Vdfl.-basis, ganz schmal und in der Draufsicht nicht sichtbar. Hfl. hellbraun bis grau, Basis aufgehellt. Diese Merkmale können einzeln oder gemein- sam variieren, so dass es Tiere gibt, die äußerlich von naumanni nicht zu trennen sind.

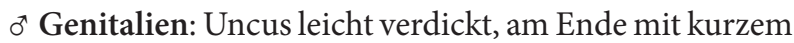
Haken. Tegumen dorsal stark verbreitert, Valve am Ende zweigeteilt, ventral zugespitzt und stärker sklerotisiert, Valve ventral mehr bauchig vorgewölbt. Innerer Fortsatz kräftig sklerotisiert, halbkreisförmig gebogen, am Ende stumpf abgerundet, ventral mit einer schmalen Reihe kurzer Haare, Basis der Fortsätze durch eine streifenförmige Sklerotisierung verbunden. Aedoeagus kurz.

ㅇ Genitalien (möglicherweise naumanni): 8. Segment breit sklerotisiert, ventral häutig. Ostium in der Intersegmentalhaut, kaudal mit einer flächigen Sklerotisierung. Ductus bursae etwa so lang wie das Corpus bursae mit einem langen schmalen, rinnenförmigen Antrum, welches in das kugelförmige, fast vollständig bedornte Corpus bursae hineinreicht. Signum klein, dessen Umfeld ebenfalls unbedornt.

Differentialdiagnose: Im Habitus tendenziell kleiner und Färbung dunkler als naumanni. Uncus schmaler, inneren Fortsätze der Valve halbrund und am Ende stumpf, Antrum im Ductus schmal.

Derivatio nominis: Benannt im Gedenken an Henri Hoppe (02.06.1964-14.10.2011), Lepidopterologe und Freund.

\section{Nanna griseata KüHNE, 2007}

Tafeln 1, 5

Nanna griseata KüHNE, 2007: 355

Material: HT, ơ, „coll. Museum Tervuren/ Côte d'Ivoire: Forêt/ classée Bossematié/ Code H2 30/09/96/ m 650 du transect 5/ Ugo Dall'Asta“, [MRAC]; PT: $80^{\star}$, dito, 19.ix.02.x.1996, Ugo Dall'Asta; 1 đ`, 1 우, Côte d'Ivoire, Forêt classée Bossematié, 11.x.1993; [MRAC, MfN, LKP]; CI: 5 ơ, 1 ㅇ, Forêt classée Bossematié, 1., 2.10.1996, U. Dall'Asta; 3 ơ, Forêt classée Bossematié, 19., 27.9.1996, U. Dall'Asta; NG: $2 o^{\star}, 5$ ㅇ, Ikom, 21.-24.12.1970, Dr. Politzar; 1 o $^{\star}, 2$ ㅇ, Danane, 12.12.80, 4.3.1981, Politzar; $1 \sigma^{\star}, 1$ ㅇ, Tai Nat. Park, 11.12.1982, 23.10.84, Dr. Politzar; $2 \sigma^{*}$, Ore, 27.12.1970, Dr. Politzar; CM: 1 ơ, Edea, 9.v.1070, C. Herbulot, CI: $1 \sigma^{\star}$, San Pedro, 13.-16.12.1976, Dr. Politzar; $1 \sigma^{\top}$, Daloa, 21.10.76, Dr. Politzar; BF: $1 \sigma^{\star}$, Bobo, 18.ix.1976, Dr. Politzar; [MRAC, ZSM, LKP].

Habitus: Spannweite: Männchen 30-38 mm, Weibchen 30-36 mm, diese im Habitus etwas runder erscheinend als die Männchen. Grundfärbung der Vdfl. grau bis hellgrau, Hfl. manchmal gleichfarbig, aber meist etwas heller, insbesondere wohl in trockeneren Gebieten, zum Saum hin meist dunkler als an der Wurzel. Kopf und 
vorderer Thorax dunkel, übriger Thorax grau, Abdomen gelb beschuppt.

$0^{\top}$ Genitalien: Uncus schmal, mit hakenförmiger Spitze, diese manchmal im GP aufgespalten und dadurch als gegabeltes Uncusende erscheinend (Artefakt). Valve zweigeteilt, dorsaler Teil gleichmäßig sklerotisiert, distal verbreitert, ventraler Valventeil mittig verbreitert, dann stark verjüngt, kräftiger sklerotisiert, am Ende mit Spitze, insgesamt nur leicht gebogen. Der innere Fortsatz kurz, kräftiger, leicht gebogen, dicht bedornt und mit kurzer Spitze. Am Aedoeagus der Fortsatz schmal. Vesica fast vollständig mit kleinen Dornen besetzt.

q Genitalien: Ostium tief in das 7. Segment eingesenkt und dessen Ränder schräg an der Einsenkung umgeschlagen. Diese Struktur erscheint im GP als breite, randlich geriffelte, stark sklerotisierte Einfassung, die nach kaudal auseinander läuft. Ductus bursae breit, ansatzlos in das Corpus bursae übergehend. Im Ductus bursae befindet sich ein Antrum, welches sich proximal auf etwa $2 / 3$ verjüngt. Das Corpus bursae ist innen stark bedornt, Signum mit unbedornter Umgebung.

Differentialdiagnose: Männchen leicht durch ihre Größe von naumanni, colonoides und diplisticta, im männlichen Genital eindeutig durch den kürzeren inneren Valvenfortsatz zu unterscheiden. Weibchen durch die Form des Antrum von hoppei sp. nov. leicht zu trennen.

\section{Nanna distyi sp. nov.}

Tafeln 1, 5

Material: HT: $0^{\star}$, „Musèe du Congo/Lusambo/ 10-XI1949/Dr. M. Fontaine" Präp. 455, [MRAC].

PT: 1 o , „Cameroon“, „Sjöstedt“, Präp. 794, [NHRS].

Weiteres Material, nicht zur Typenserie gehörend: 1 우, Uele: Paulis, 15.II.1957, M. Fontaine, präp. 594.

Habitus: Spannweite Männchen $36-38 \mathrm{~mm}$, Weibchen $48 \mathrm{~mm}$, Vdfl. des einen Männchens grau, des anderen fast weiß, ohne dunklen Costalrand, Punkte kräftig, der Analpunkt deutlicher, Hfl. fast weiß, Weibchen gräulich. Kopf und Kragen gelb, übriger Thorax in der Farbe der Vdfl.; Vordertibien innen verdunkelt. Fühler fadenförmig, beborstet.

$0^{\top}$ Genitalien: Uncus schmal, Tegumen insgesamt fast gleich breit, lateral gewölbt. Valven bis etwa $1 / 3$ geteilt, dorsaler Teil gleichmäßig sklerotisiert und gestreckt, distal nicht verbreitert, kaum abgerundet. Ventraler Teil distal auch zu einem stärker sklerotisierten Fortsatz verjüngt, dieser dicht bezahnt, innerer Fortsatz kurz, rund und an der Basis rechtwinklig gebogen, dicht bezahnt, mit kleiner Spitze. Juxta fast doppelt so lang wie breit. Aedoeagus proximal konisch, distaler Fortsatz kurz, am Ende abgerundet, Vesica fast vollständig mit kleinen Dornen besetzt.
† Genitalien: Apophysen des 9. Segments dünn und lang, am 8. Segment kurz. Dieses gleichmäßig breit, ventral vor dem Ostium häutig, hier kaudal mit umgeschlagenen breiten, abgerundeten Ecken. Das 7. Segment gleichmäßig sklerotisiert und in die Intersegmentalmembran übergehend, hier der kaudale Rand nur mittig vor dem Ostium etwas schwächer sklerotisiert, aber nicht ein- oder umgeschlagen wie bei vielen anderen Arten. Ductus bursae mittellang, Corpus oval, lateral zum Ductus bursae hin ein kräftiges Signum, die Bedornung im Corpus bursae gegenüber dem Signum beginnend und nach kranial dann das ganze Corpus bursae einnehmend.

Differentialdiagnose: Im Habitus durch die weißlichen Hfl. tendenziell, im männlichen Genital sicher durch den rechtwinkligen inneren Valvenfortsatz von griseata $\mathrm{zu}$ trennen. Von griseoides sp. nov. nur anhand des Vorhandenseins des inneren Valvenfortsatzes zu unterscheiden.

Derivatio nominis: Gewidmet meinem Freund und häufigen Gastgeber in Westafrika, dem deutschen Entwicklungshelfer Thomas Diestelhorst (17.07.196331.08.2008), Spitzname Disty.

Nanna collinsii KüHNE, 2007

Tafeln 1, 6

Nanna collinsii KüHNE, 2007: 355

Material: HT, ơ, „coll. Mus. Congo/ Uele: Paulis/ 14-IV1956/ Dr. M. Fontaine“, Präp.: 451 [MRAC]; PT: $2 \sigma^{\star}$, Uele: Paulis, 1956-59, Dr M. Fontaine; $8 \sigma^{\star}$, Kenya, Kakamega Forest, verschiedene Standorte, iv., x.2001, leg. Dr. U. Dall'Asta, L. Kühne; [MRAC, MfN, SMNS, LKP]; $20^{\star}$, Zaire, Irangi, Stat. Luoho, 3.-8.3.1984, Burmeister, Fuchs, Kühbandner (Präp. 577); $10^{\top}$ Zaire: Kibali-Ituri, Beui, 21.VII.1952, J. Hecq; 1 o $^{\star}$, Kenia, Kakamega Forest, 19/04/2001, U. Dall'Asta; 1 \& , Cameroon, „Sjöstedt“, Präp. 440 [NHRS]; DRC: 1 우 Kafakumba, X.1930, G. F. Overleat, [LKP]; 1 ㅇ, Katako-Kombe, 21.iii.1952, Dr. M. Fontaine, [MRAC]; CM: 1 ㅇ, Bitje, Ja River, OctNov. 1912, Rosenberg, [MRAC].

Der Urbeschreibung lag überwiegend Material aus Ostafrika zugrunde. Daneben existierte seinerzeit von gleichen Fundorten Material von eningae albifrons. Die scheinbare Variabilität des Corpus bursae in den GP in Abhängigkeit von Torsion, Färbung und Begattung und die unvollständige Aufklärung der Verhältnisse in der eningae-Gruppe, führten damals dazu, dass fälschlicherweise "weiße" eningae-Weibchen als collinsii angesehen wurden.

Diese weiblichen Paratypen sind nun wie folgt neu $\mathrm{zu}$ ordnen: zu eningae albifrons: 1 , , PT Kenya, Kakamega Forest, zu eningae eningae: 2 우, Uele: Paulis, 1956-59, Dr. M. Fontaine 
Habitus: Spannweite der Männchen 39-45 mm, Weibchen größer, 50-53 mm. Flügel weiß, etwas glänzend, ältere Exemplare dunkeln nach und ähneln dann mehr eningae. Costalrand im basalen Viertel schmal, schwarz. Punkte im Vdfl. deutlich, schwarz, der Analpunkt oft etwas länglich und leicht nach innen versetzt, Costalpunkt mit halbem Durchmesser von der Costa entfernt. Kopf und Kragen gelb bis gelborange, übriger Thorax und Ansatz des Abdomen weißgrau, Abdomen dann fast gleichmäßig gelb. Vorderbeine am Femur-TibiaGelenk schwarz, sonst mehr oder weniger verdunkelt, Mittel- und Hinterbeine fast weiß. Fühler dorsal weiß beschuppt.

$\sigma^{\star}$ Genitalien: Uncus schwach gebogen, mit kleiner Spitze, Tegumen breit, Saccus nur als sklerotisierte Spange, Valve zweigeteilt, der ventrale Teil schmaler und kräftiger sklerotisiert, am Ende halbrund nach dorsal gebogen, wie bei eningae, aber die Valve insgesamt kürzer. Ein weiterer Unterschied besteht in den kräftigen Fortsätzen des Tendons an der Valve. Diese fast so lang wie die Valve breit, mit feinen Dornen, am Ende mit deutlich verjüngter Spitze. Aedoeagus gerade; am Ende mit einer verstärkten, abgerundeten Sklerotisierung, Vesica mit einem Zähnchenfeld.

† Genitalien: Apophysen des 9. Segments lang und dünn, mehr als doppelt so lang wie die des 8. Segments. Dieses gleichmäßig breit sklerotisiert, ventral breit häutig und sekundär schwach sklerotisiert. Ostium in der Intersegmentalhaut; Ductus bursae kurz, ohne Sklerotisierung, fast übergangslos in das große, birnenförmige Corpus bursae übergehend. Dieses etwa in der Mitte lateral mit einem Signum.

Differentialdiagnose: Äußerlich durch den Abstand des Costalpunktes von der Costa und die weiß beschuppten Fühler von den äußerlich ähnlichen melanosticta, kamerunica, falcata, pia und eningae albifrons zu trennen. Im Genital von diesen schon durch die gesamte Valvenform (abpinseln) bzw. den inneren Valvenfortsatz sicher zu unterscheiden. Weibchen im Genital durch das birnenförmige Corpus bursae unterscheidbar, gegenüber eningae fehlt das Antrum.

\section{Nanna melanosticta (BetHUNE-BAKER, 1911)}

Tafeln 1, 6

Ilema melanosticta BETHUNE-BAKER, 1911: 534

Material: HT, o’, “N’Dalla Tando/N. Angola/2,700 feet/5xi-1908/Dr. W. S. Ansorge“, Präp: Arc 5673, [BMNH].

Habitus: Von der Art ist nur der Typus bekannt. Spannweite $39 \mathrm{~mm}$, Grundfarbe hell ockergrau, Punkte im Vdfl. sehr deutlich, der Analpunkt ist deutlich nach innen gesetzt. Auffällig und einmalig für die Gattung sind die stark gekämmten Fühler (vgl. magna Birket-Smith). $\sigma^{*}$ Genitalien: Valve lang gestreckt und leicht zweigeteilt, der dorsale Teil distal leicht verbreitert, der ventrale Fortsatz ist viel robuster gebaut als bei den verwandten Arten, am Ende fast rechtwinklig aufwärts gebogen, stark verjüngt, mit zahlreichen Dornen. Besonders markant ist eine lappenartige Sklerotisierung im distalen Drittel des ventralen Valventeils. Diese Struktur wird bei keiner anderen Art gefunden. Aedoeagus kurz, sklerotisierter Fortsatz kurz, Vesica mit kleinem Dornenfeld.

\section{i Genitalien: Unbekannt}

Differentialdiagnose: Die gekämmten Fühler, im männlichen Genital die lappenartige Sklerotisierung am ventralen Fortsatz der Valve sind die Charakteristika dieser Art gegenüber den anderen Taxa.

\section{Nanna pia (StRAND, 1912), stat. rev.}

Tafeln 2, 6

Eilema eningae cum var. pia StrAnd, 1912: 99 (nach Artikel 45.6.4 ICZN verfügbar)

= Nanna magna Birket-SMith, 1965: 46, Abb. 34, 35, syn. nov.

Material: Lectotypus von pia (hier designiert): $\sigma^{*}$, „N-Kamerun/ Joh.-Albrechtshöhe/----/L. Conradt S.“, Präp: 205/2004, [MfN].

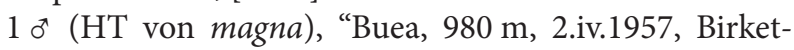
Smith", [NHRS];

CM: 1 o $^{\star}$ Edea, 9.iv.1970, C. Herbulot; 1 o $^{\star}$, Bitje, Ja River, Oct.-Nov.1912; CI: 1 o, Danane, 23.-24.4.1982, Dr. Politzar; $1 \sigma^{\star}$, Danane, 4.3.1981, Dr. Politzar; 1 ơ, Danane, 23.-24.4.1982, Dr. Politzar; $2 \sigma^{*}$, Lamto, 30.9.1970; 1 o $^{\star}, 1$ ㅇ․ Daloa, 21.X.1976, Dr. Politzar; $1 o^{\star}$, Abidjan, 13.10.1959, Birket-Smith; GH: $20^{*}$, Opro River Res., 3.-8.4.1966, S. Endrödi-Younga; NG: 1 \% , Jemaa, 15.IX.1971, H. Politzar; 1 o, Don de M. Trolli; [MRAC, MfN, LKP, NHRS].

Wie bereits oben angemerkt, sind einige Angaben von Birket-Smith nicht verifizierbar. Dies betrifft im Fall von magna das diagnostisch eindeutige Merkmal Fühler. Birket-Smith (1965: 37, Fig. 25 A) gibt an, dass die Fühler von magna bipectinat sind. Dem Typus von magna fehlen die Fühler komplett. Andere abweichende Merkmale zu pia sind nicht vorhanden. Das Merkmal „Fühler bipectinat“ stufe ich als eine der vielen Verwechslungen bei Birket-Smith ein. Wahrscheinlich wurde seinerzeit die ursprüngliche Zeichnung falsch beschriftet, denn außer melanosticta Bethune-Baker besitzt kein einziges der vorliegenden Tiere und somit kein Vertreter der Gattung auch nur andeutungsweise einen solchen Fühlerbau. Birket-Smith hatte den Typus von melanosticta untersucht, möglicherweise rührt die Verwechselung bei der Wiedergabe aus dieser Untersuchung. Somit ist das einzige für magna diagnostisch verwertbare Merkmal nicht überprüfbar und muss aufgrund des vorliegenden Materials als falsch angesehen werden. Alle anderen morphologischen Fakten 
belegen die Synonymie zu pia, sie wird hiermit als Synonym eingezogen.

Habitus: Spannweite: Männchen 34-42 mm, Weibchen $45 \mathrm{~mm}$, Grundfarbe wie bei eningae eningae gelblich grauweiß, Vdfl. etwas dunkler als Hfl., Saum schwach verdunkelt, Punkte deutlich, Kopf meist gelb, gelegentlich auch der Ansatz der Tegulae, Thorax in der Farbe der Vdfl., Abdomen zum Ende hin gelblich, Fühler etwas dicker als bei den eningae, gleichartig geborstet. Vorderbeine verdunkelt.

o Genitalien: Uncus mittellang, Valve über die Mitte geteilt, dorsaler Valventeil schlank, am Ende etwas breiter und leicht nach dorsal gezogen, ventraler Valventeil zugespitzt, aufwärts gebogen, stärker sklerotisiert. Aedoeagus kurz, etwas konisch, sklerotisierter Fortsatz leicht gebogen.

ㅇ Genitalien: Apophysen des 8. Segments kurz, etwa nur halb so lang wie die Apophysen am 9. Segment. 8. Segment breit sklerotisiert, ventral kaudal mit abgerundeten Ecken, kranial vor dem Ostium plattenförmig sklerotisiert. Ductus bursae kurz und wohl ohne Antrum, das ovale Corpus bursae nicht abgesetzt, innen mit ganz kleinen Dornen und einem Signum. Abgang des Ductus seminalis am Übergang zwischen Ductus und Corpus bursae.

Differentialdiagnose: Habituell schwer von eningae und kamerunica zu trennen. Uncus tendenziell etwas schmaler und länger als bei eningae, dorsaler Valventeil mehr gerade, in der Mitte etwas dünner, dadurch gestreckter erscheinend. Dem Weibchen fehlt im Vergleich offensichtlich das Antrum, das kann aber nur durch die Untersuchung weiterer Weibchen abgesichert werden. Alle anderen ähnlichen Arten im Genitalbau leicht zu unterscheiden.

\section{Nanna eningae eningae (PLÖTZ, 1880)}

Tafeln 2,7

Gnophria? eningae Plötz, 1880a: 80

= Nanna montana Birket-SMith, 1965: 44 (synonymisiert KüHNE 2005a)

Im Rahmen der Revision mussten die nahe stehenden, außerordentlich schwierig zu fassenden Taxa entgegen früherer Schlussfolgerungen (KüHNE 2005a, 2007) neu geordnet werden.

Ein wichtiger Schlüssel hierfür war die nunmehr sichere Identifizierung des Typenfundortes von eningae, denn die vorherige Verortung in Westafrika hatte zu falschen Schlussfolgerungen bezüglich der Taxa magna, montana und pia geführt. Der Typenfundort Eningo ist keine Ortsangabe, sondern bezieht sich auf eine ethnische Gruppe bzw. ein kleines Reich in der Landschaft Loango (Gabun) am Ogowe(Ogooué)-Fluss. Der Sammler Prof. Reinhold
Buchholz fuhr damals von Cap Lopez (Port Gentil) flussaufwärs und gelangte gemäß seinem Tagebuchauszug (vgl. Heinersdorff, 1880) über den Orongo und Ovanga in das Eningagebiet zwischen Ogowe und dem Elivasee (= Silesee). Dies ist ein See zwischen dem Jonanga- und Anengesee, der nicht mehr klar identifizierbar ist.

Eine weitere gute Grundlage für die Klärung und Herausarbeitung diagnositisch verwertbarer Merkmale boten die langen Serien, die im Rahmen dieser Untersuchung zur Verfügung standen.

Die Art bildet in der Flügelzeichnung und -färbung geografisch gut abgrenzbare Unterarten aus, die nur gelegentlich Überschneidungen besitzen. Allen gemeinsam ist, dass ältere Tiere in Sammlungen nachdunkeln bzw. bei hoher Luftfeuchtigkeit ihre Färbung vertiefen. An den Genitalarmaturen sind keine diagnostisch verwertbaren Merkmale zur sicheren Unterscheidung der Taxa zu ermitteln. Diese Tatsachen führen zu der Annahme, dass der Speziationsprozess hier noch nicht abgeschlossen ist. Dagegen unterscheiden sich die nahe verwandten Taxa pia STRAND, tanzania sp. nov. und luteata sp. nov. anhand der Genitalmorphologie und ihrer Verbreitung. In der Gesamtschau sind alle von BiRket-SMith (1965) in dieser Gruppe neu beschriebenen Taxa (s.o.) nicht als valid zu betrachten. Die von ihm angegebenen Unterscheidungsmerkmale an den Fühlern (Fig. 25, ebenda) sind nicht verifizierbar bzw. treffen nicht $\mathrm{zu}$ (vgl. Bemerkung bei pia). Hinzu kommt der von ihm selbst erwähnte schlechte Erhaltungszustand seiner untersuchten Falter.

Material: HT eningae, o, "W.-Afrika/Eningo/13. VI.1875/leg. R. Buchholz", [coll. Universität Greifswald]; HT montana, o*, "Kamerun, 2.iv.1957, Birket-Smith", [NHRS]; DRC: 1 क Luki-Mayumbe N. R., 5.IV.2006, J. de Prins; $19,2.1935$, A. Bal; 2 , Paulis [Uele], 1956-59, Dr. M. Fontaine; Eala, VI-1936, J. Ghesquière; $1 \sigma^{\star}$, Tungudu, 13.VI.1953, P. Vantschuybroeck \& J. Kekenbosch; $20^{\star}$, Watalinga, 24.VII.1957, P. Vantschuybroeck; $2 \sigma^{\star}$, Katako-Kombe, 29.VII., 13.XI.1952, Dr. M. Fontaine; 4 ơ 1 ㅇ, Paulis [Uele], IX.1957-29. VI.1960, Dr. M. Fontaine; 1 ơ, Kilo, 1955, R. Andry; AO: 6 ơ, Quicolungo, 18., 22.X.1957, Gerd Heinrich; CM: $1 \mathrm{o}^{\star}$, Bitje, Ja River, Oct.-Nov.1912; [MRAC, LKP].

Habitus: Spannweite: Männchen 32-40 mm, Weibchen 45-50 mm, Grundfarbe gelblich grauweiß, hell, leicht glänzend. Vdfl. etwas dunkler, der dunkle Costalrand schmal und nur an der Wurzel vorhanden, Punkte bei den Männchen mittel bis schwach ausgebildet, der Analpunkt schwächer, leicht bis stark nach innen versetzt, bei den Weibchen variierend, oft nach außen stehend und der Costalpunkt manchmal in den Rand fließend. Kopf, Thorax und 1. Abdominalsegment etwa in der Farbe der Vdfl., mit einem stahlgrauen Farbstich, Stirn gelb! beschuppt, Fühler ohne Auffälligkeiten, Abdomen gelb, zum Ende etwas kräftiger in der Färbung. Vorder- und Mittelbeine dunkel (variierend), Hinterbeine gelb. 
Uncus kurz, am Ende mit Haken, Tegumen nur lateral verschmälert, Valven etwa bis zur Mitte zweigeteilt, dorsaler Valventeil am Ende rundlich verbreitert, aufwärts gebogen, ventraler Valventeil dünn, zugespitzt, stark sklerotisiert und aufwärts gebogen, ganz kurz behaart. Juxta breit, ventrale Arme kurz, Aedoeagus mittellang, distal mit einer sklerotisierten Spange, Vesica mit Dornenfeld.

i Genitalien: Apophysen am 8. Segment kurz, etwa nur halb so lang wie Apophysen am 9. Segment. 8. Segment breit sklerotisiert, ventral kaudal mit abgerundeten, vorgezogenen Ecken, kranial vor dem Ostium mit einer nach innen gewölbten sklerotisierten Platte. Ductus bursae kurz und breit, mit einem zarten kurzen Antrum, das ovale Corpus bursae mit kleinen Dornen und kleinem Signum, wenig abgesetzt. Abgang des Ductus seminalis am Übergang zwischen Ductus und Corpus bursae. Bei stärker gefärbten Präparaten (Chlorazol black) sind Antrum und Signum nicht mehr wahrnehmbar.

Differentialdiagnose: Punkte im Vdfl. eher schwächer als bei pia und Färbung im Vergleich leicht gelblicher, der Kopf und Fühleransatz gelblich gefärbt. Uncus gegenüber pia eher kürzer, dorsaler Valventeil am Ende mehr verbreitert und insgesamt mehr nach dorsal gebogen, weibliche Genitalien mit Antrum im Ductus bursae.

Unterschiede der Männchen und Weibchen zur äußerlich ähnlichen collinsii: Etwas dunkler, Costalpunkt direkt an der Costa, bei collinsii mit halber Punktbreite Abstand. Im Genitalbau sicher zu trennen: bei den Männchen fehlt der innere Valvenfortsatz von collinsii, Weibchen mit Antrum, welches bei collinsii fehlt. Die Verbreitung umrandet das Kongobecken von Kamerun über Nordangola bis zum westlichen Uganda.

\section{Nanna eningae albifrons ssp. nov.}

Tafeln 2, 7

Material: HT: ơ, "Kenya-Western / Kakamega Forest N. R. / Udo's campsite 1600 m / 16.ix.2001 (Lichtfalle) / 0.21,08N 34.51,57E / leg. L. Kühne" [LKP, später MfN, may be.] PT: 26 ơ, 2 + , ebenda, 1997-2003 (Eitschberger, Bauer \& Traub, U. Dall'Asta, L. Kühne, J. Holstein); $30^{*}$, Rwanda, Butare, 1974-77, Turlin; 2 o $^{\star}$, B[?], 1973, 1979; 4 o , KibaliIturi: Nioka, 1953, J. Hecq; 9 ơ, Kivu: Rwankwi, 1947-48, J. V. Leroy; [MRAC, ZSM, MfN, LKP].

Beschreibung, Habitus: Männchen größer als eningae, 38-45 mm, Weibchen 50-54 mm, Vdfl. und Hfl. fast weiß, ganz leicht gelbweiß. Punkte oft nicht vorhanden oder sehr klein, selten deutlich wie bei eningae. Kopf, Thorax und 1. Abdominalsegment in der Farbe der Vdfl., Abdomen zum Ende hin zunehmend gelblicher. Fühler dunkel, Vorder- und Mittelbeine individuell verdunkelt, Hinterbeine meist gelblich.

đ Genitalien: Von eningae eningae nicht unterscheidbar.
+ Genitalien: Von eningae eningae nicht unterscheidbar.

Differentialdiagnose: Die fast weiße Färbung und das häufige Fehlen der Punkte ermöglichen eine leichte Unterscheidung von den anderen ssp., Kopf in Draufsicht ohne gelbe Stirn.

Unterschiede zur äußerlich ähnlichen collinsii: Punkte viel schwächer, Costalpunkt direkt an der Costa, bei collinsii mit halber Punktbreite Abstand. Im Genital fehlt den Männchen der innere Valvenfortsatz von collinsii, bei den Weibchen ist der Ductus bursae breiter, dadurch erscheint das Corpus bursae fast gleich dick. Die Unterart ist in den Regenwaldfragmenten im ostafrikanischen Grabensystem verbreitet.

Derivatio nominis: Abgeleitet von weiße (lat. = albus) Stirn (lat. = frons), dem Unterscheidungsmerkmal gegenüber den anderen ssp.

\section{Nanna eningae subflava ssp. nov.}

Tafeln 2, 7

Material: HT: ${ }^{\star}$, „,coll. Mus. Congo / Uele: Paulis/ 18-VII1958/ Dr M. Fontaine“, [MRAC].

PT: 4 ơ 5 ㅇ, DRC, Eala, 1935-38, J. Gesquière; 4 ơ, Sankuru: Katako-Kombe, 1952-53, M. Fontaine; $50^{\star \top}$, Uele: Paulis, 1954-58, M. Fontaine; 1 ㅇ, Kamerun, Bitye, Rosenberg; $50^{\star}$, Semliki, route Watalinga, 1957, P. Vanschuytbroeck; 1 q , Ituri: Nioka, 1953, J. Hecq; $10^{\top}$, Elisabethville, 1936, Ch. Seydel; [MRAC, MfN, LKP].

Beschreibung, Habitus: Größe wie eningae eningae, Grundfarbe mehr ockergelb, Vdfl. auch etwas dunkler als Hfl. Punkte deutlich, bei den Weibchen gelegentlich der Analpunkt ganz fehlend, dunkler Costalrand etwas länger und deutlicher. Stirn bzw. Kopf gelb, Verdunkelung der Beine variierend.

$0^{\star}$ Genitalien: Von eningae eningae nicht unterscheidbar.

+ Genitalien: Von eningae eningae nicht unterscheidbar.

Differentialdiagnose: Tiere insgesamt mehr beige und Punkte kräftiger als bei pia, eningae eningae und eningae albifrons; Unterschiede im Genital zu pia siehe bei eningae eningae. Im zentralen Kongobecken bis zum westlichen Rand des ostafrikanischen Grabensystems verbreitet.

Derivatio nominis: Abgeleitet von der ockergelben (lat. gelb = flavus) Grundfarbe der Adulti.

\section{Nanna tanzaniae sp. nov.}

Tafeln 2, 8

Material: HT: † „Tansania/Mpanda/Sibwesa/3.1.1962/ leg. J. Kielland“ Präp. 798, [ZSM].

PT: $20^{\star}$, Tanganjika, Bukoba, IX-X.1964, J. Scheven (Präp. 804, 570), [ZSM, LKP]. 
Habitus: Spannweite 37-40 mm, kein Geschlechtsdimorphismus. Vdfl. blassgelb, Hfl. ein wenig heller, der dunkle Costalrand dünn, Punkte schwarzbraun. Kopf und Thorax grau bis gelblich, Abdomen gelb, Schienen dunkel.

${ }^{\star}$ Genitalien: Uncus normal gebaut, Tegumen fast gleich breit. Valven bis etwa zur Hälfte geteilt, dorsale Teile distal verbreitert und abgerundet, hier aufwärts gebogen, ventrale Teile distal verschmälert, stärker sklerotisiert und halbrund nach dorsal gebogen. Valve ohne inneren Fortsatz, Juxta als abgerundete, dreieckige Platte, ventral eingebuchtet. Aedoeagus kurz, basal etwas zugespitzt, sklerotisierter Fortsatz fast so lang wie der Aedoeagus, gebogen, am Ende abgerundet. Vesica mit kleinem Dornenfeld.

+ Genitalien: Apophysen lang, ventral am 8. Segment eine fast quadratisch sklerotisierte Platte, vor dem Ostium eine dreieckige Sklerotisierung, 7.Segment leicht umgeschlagen, Rand nur rauh, leicht ausgebuchtet. Ductus bursae kurz und breit, vollständig vom Antrum ausgefüllt, dieses schräg in das ovale Corpus bursae hineinziehend. Dieses bis auf einen Hof um das Signum innen mit kleinen Dornen ausgekleidet.

Differentialdiagnose: Etwas größer und blasser als die westliche Schwesternart luteolata sp. nov. Die sklerotisierte Platte kaudal vom Ostium mehr quadratisch und nicht so breit wie bei dieser, Antrum größer. Gut kenntlich am kurzen Aedoeagus.

Derivatio nominis: Abgeleitet von Fundland, Tanzania.

\section{Nanna luteolata sp. nov.}

Tafeln 2, 8

Material: HT: ㅇ, „Nigeria / Kaduna / 28.VII.1971/leg. H. Politzar" [ZSM].

PT: 3 ㅇ, ebenda, 1970, 1971, H. Politzar (Präp. 571); 우, Nimba (Guinea), [19]51, Lamotte \& Roy; 1 ㅇ, Lamto, 14.12.68, Gmiere; 1 + , Obervolta, Bobo Dioulasso, 1980, Dr. Politzar; [ZSM, MfN, LKP].

Habitus: Spannweite 34-36 mm, Vdfl. hell goldgelb, Hfl. etwas heller und ein wenig mehr grau, Costalrand nicht verdunkelt, Punkte schwarzbraun, Costalpunkt als kurzer Strich, Kopf, Thorax und Abdomen gelblich, lediglich Thorax und basaler Abdomenfleck mehr grau. Beine distal dunkel.

\section{o Genitalien: Unbekannt.}

† Genitalien: Apophysen dünn, länger als bei den anderen Arten. 8. Segment ventral mit einer breiten, kaudal eckigen Platte. 7. Segment kaudal umgeschlagen, Rand geriffelt, in der Mitte ganz leicht vorgezogen. Am Grund dieser tiefen Einsenkung das Ostium. Ductus bursae kurz, breit mit einem schrägen, breiten Antrum, ansatzlos in das länglich ovale Corpus bursae übergehend. Dieses in der Mitte leicht eingeschnürt, kaudal mit einer Ausstülpung und dichter mit Dornen ausgekleidet als kranial. Ein Signum in einem dornenfreien Bereich.

Differentialdiagnose: Leicht anhand der gelben Vdfl. von allen anderen Arten zu trennen.

Derivatio nominis: Abgeleitet von luteus (lat. = goldgelb) aufgrund der Flügelfarbe.

\section{Nanna griseoides sp. nov.}

Tafeln 2, 8

Material: HT: o", "Cameroun/Mt. Cameroun/26.XII. 1991/leg. Rautenstrauch“ Präp. 456 [NKMD].

PT: 3 đ`, Mt. Cameroun: Bonenza, 18.1.1989, Präp. 661, 565; ơ, ebenda, 14.1.1989, Präp. 566; 2 ๙, Camerun, Sjöstedt, Präp. 443; [MRAC, NHRS, LKP].

Beschreibung, Habitus: Spannweite: 34-38 mm, Vdfl. schmal, Apex leicht vorgezogen, gräulich, Punkte variabel, gelegentlich kräftig ausgebildet, Analpunkt leicht nach innen versetzt, dunkler Costalrand fehlend, Hfl. einfarbig hell weiß-gelblich. Körper gleichmäßig schmutzig gelb beschuppt. Fühler kurz bewimpert, Beine gelblich.

o Genitalien: Uncus kurz, basal etwas verdickt, Tegumen dorsal stark verbreitert, Vinculum als kräftige Spange, ein wenig eckig erscheinend, Valven fast bis zur Hälfte geteilt, dorsaler Teil am Ende mehr rundlich verbreitert, ventraler Teil am Ende zugespitzt, hier stärker sklerotisiert und aufwärts gebogen. Juxta gleichmäßig sklerotisiert, ventral mit zwei breiten Armen. Aedoeagus kurz, kranial zugespitzt, der distale Fortsatz leicht gewellt, Vesica mit kleinen Dornenfeld.

\section{ㅇ Genitalien: Unbekannt}

Differentialdiagnose: In der Färbung ähnlich griseata, aber nicht so grau, etwas kleiner und anhand der fehlenden inneren Valvenfortsätze von dieser sicher unterscheidbar. Abgeflogene Tiere anhand des verbreiterten dorsalen Valvenfortsatzes und dem kürzeren Uncus von Arten der eningae-Gruppe leicht zu trennen. Auch gut kenntlich am gleichartig gelb gefärbten Körper, weil im Gegensatz dazu bei den anderen Arten der Thorax und die Flügel von gleicher Färbung sind.

Derivatio nominis: Abgeleitet von der Ähnlichkeit zu N. griseata. 


\section{Nanna kamerunica KüHNE, 2007}

Tafeln 2, 9

Nanna kamerunica KüHNE, 2007: 355

Material: HT, ơ, „Cameroun/ Mt. Cameroon/ 28.XII. 1991/ leg. Rautenstrauch/ Ankauf 1999, NKMD”, Präp.: 439; [NKMD]; PT: 3 o , dito; [NKMD; LKP].

Habitus: Spannweite 40-42 mm, Vdfl. schmutzig weiß mit den zwei typischen dunklen Punkten, wobei der Punkt an der Costa im Vergleich zu den anderen Arten schwach strichförmig ist. Hfl. etwas heller. Thorax in der Farbe der Vdfl., Kopf ähnlich, nur die Stirn mit gelben Schuppen, Abdomen gelblich beschuppt. Vordertibien schwarz.

$0^{\top}$ Genitalien: Tegumen breit, bildet mit dem Vinculum einen fast runden Kreis. Sofort auffällig sind im Vergleich zu den anderen Arten die breiten Valven. Valve tief geteilt, der ventrale Teil der Valve mehr geschwungen und der dorsale Teil fast dreimal so breit wie bei den anderen Arten. Das spitze Ende des Sacculus gleichmäßig gebogen, kräftig. Juxta breit sklerotisiert, die basalen Arme gebogen. Der distale, sklerotisierte Fortsatz am Ende des Aedoeagus so stark aufgetrieben, das er breiter als der Aedoeagus ist, etwas eingerollt, außen mit einem dichten Zähnchenfeld. Vesica mit mehreren Dornenfeldern.

\section{† Genitalien: Unbekannt.}

Differentialdiagnose: Nur durch Genitaluntersuchung von den habituell ähnlichen Arten anhand der breiten Valven sicher zu unterscheiden.

\section{Nanna falcata sp. nov.}

Tafeln 2, 9

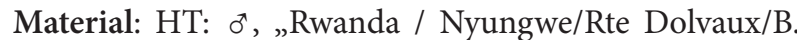
Turlin/ 2000* 19-X-74“, Präp. 663, [ZSM].

PT: 1 ㅇ, dito, Präp. 664; 3 o $^{\star}$, Forèt Nyungwe, Route Delvaux km 16, 15.4.1977; 1 ㅇ, Nyungwe, ebenda, 12-X74; [ZSM, LKP].

Beschreibung, Habitus: Spannweite: Männchen 43-45 mm, Weibchen $50 \mathrm{~mm}$, Vdfl. und Hfl. weißlich, Punkte kräftig schwarz, dunkler Costalrand max. 1/3 der Vdfl.-länge. Nur der Kopf gelborange beschuppt, Thorax vollständig schmutzig weiß, Abdomen gelborange, zum Ende hin kräftiger gefärbt. Fühler der Männchen bewimpert, basale Hälfte dorsal dunkel beschuppt, Vorderbeine gleichfalls dunkel beschuppt, die übrigen Beine gelb.

ơ Genitalien: Uncus lang, dünn, Tegumen fast gleich breit, Valven etwa bis $1 / 3$ zweigeteilt, dorsaler Teil distal verbreitert und auffällig gleichmäßig sklerotisiert, ventraler Teil distal hippenförmig gebogen, spitz, kräftig sklerotisiert, hier distal dicht behaart. Aedoeagus mittellang, kranial verjüngt, der sklerotisierte Fortsatz distal knopfartig, Vesica mit kleinen Dornenfeldern.

o Genitalien: Apophysen des 9. Segments fast doppelt so lang wie die des 8. Segments. Ventral von der Afteröffnung eine paarige, leicht vorgewölbte Sklerotisierung. 8. Segment als breiter Ring sklerotisiert, lateral teilweise und ventral komplett häutig. Ostium frei in der Intersegmentalhaut, Ductus bursae gedrungen, das Corpus bursae oval, mit geriffelter Oberfläche, keine Zähnchen oder ein Signum erkennbar.

Differentialdiagnose: Männchen nur anhand der hippenartigen Form des ventralen Valventeils sicher zu diagnostizieren. Bei den Weibchen ist die fehlende innere Bezahnung des Corpus bursae typisch.

Derivatio nominis: Abgeleitet von falx (lat. = Hippe, Sense) aufgrund der Form des ventralen Valventeils.

\section{Danksagung}

Für die Geduld bei den Ausleihen gilt mein Dank den Herren Prof. Gerd Müller-Motzfeld ( $\dagger$ ) und Peter Michalik (Universität Greifswald), Axel Hausmann und Ulf Buchsbaum (München), Ugo Dall'Asta (Tervuren), Timm Karisch (Dessau), Bert Gustafson (Stockholm) und Wolfram Mey (Berlin). Ich danke Martin Krüger (Pretoria) für die wertvollen Diskussionen und die Möglichkeit, einen Einblick in die Sammlungsaufstellung für seine Lithosiini-Revision zu erhalten, Sylke Frahnert (Berlin) für die wertvollen Hinweise zum Fundort Gunnal und bzgl. Wiliam Ansorge, sowie meiner Familie.

\section{Literatur}

Bendib, A. \& Minet, J. 1999: Lithosiine main lineages and their posible interrelationships. I. - Defination of new or resurrected tribes (Lepidoptera: Arctiidae). - Annales de la Societae Entomologique de France. (N.S.), 35 (3-4): 241-263.

Bethune-Baker, G. T. 1911: Descriptions of new Species of Lepidoptera from Tropical Africa. - Annals and Magazine of Natural History, (Ser. 8) 8: 506-542.

Birket-Smith, J. 1965: A revision of the West African Eilemic Moths, based on the Male Genitalia (Lep., Arctidae, Lithosiinae). - Aritistic Printers, Addis Ababa, $163 \mathrm{~S}$.

Boulenger G. A. 1905: Descriptions of four new freshwater fishes discovered by Dr. W. J. ANsorge in Angola. - The Annals and Magazine of Natural History (Ser. 7) 15 (89): 457-459. 
Boulenger G. A. 1910: On a large collection of fishes made by Dr. J. W. Ansorge in the Quanza and Bengo Rivers, Angola. - The Annals and Magazine of Natural History (Ser. 8) 6: 537-560.

Boulenger, G. A. 1906: On some fishes from the Kwango River (Congo System) in Angola, collected by Dr. W. J. Ansorge. - Annals and Magazine of Natural History (Ser. 7) 17: 110-112.

Boulenger, G. A. 1911a: Descriptions of new freshwater fishes discovered by Dr. W. J. Ansorge in Portuguese Guinea. - Annals and Magazine of Natural History (Ser. 8) 7: 373-376.

Boulenger, G. A. 1911b: Further descriptions of new freshwater fishes discovered by Dr. W. J. Ansorge in Portuguese Guinea. - Annals and Magazine of Natural History (Ser. 8) 8: 56-57.

HeinersdorfF, C. 1880: Reinhold Buchholz' Reisen in West-Afrika nach seinen hinterlassenen Tagebüchern und Briefen, nebst einem Lebensabriss des Verstorbenen. - Leipzig: Brockhaus, 263 S.

Dean, W. R. J.; Walters, M. P. \& Dowsett, R. J. 2003: Records of birds breeding collected by Dr W. Ansorge in Angola and Gabon. - Bull. Brit. Orn. Club 123 (4): 239-250.

Dean, W. R. J.; Adams, M.; Frahnert, S. \& Milton, S. J. 2009: William John Ansorge's bird collections in Guinea-Bissau: an annotated list. - Malimbus 31: 75-108.

Durante, M. A. \& Panzera, S. 1999: I Lepidotteri del Delta del Niger. Primo Contributo. Conferma di Tesma fractifascia (Hampson, 1918) e Descrizione della Femina (Lepidoptera, Arctiidae, Lithosiinae). Lambillionea 99 (3): 439-448.

Durante, M. A. \& Panzera, S. 2001a: I Lepidotteri del Delta del Niger (Secundo contributo) (Lepidoptera, Arctiidae, Lithosiinae). - Lambillionea 101 (2): 203-215.

Durante, M. A. \& Panzera, S. 2001b: I Lepidotteri del Delta del Niger (Terzo contributo) (Lepidoptera, Arctiidae, Lithosiinae). - Lambillionea 101 (3): 465-472.

Durante, M. A. \& Panzera, S. 2002a: Pusiola unipunctana major n. ssp. della Repubblica di Guinea (Lepidoptera, Arctiidae, Lithosiinae). - Lambilionea 102 (1): 88-90.

Durante, M. A. \& Panzera, S. 2002b: I Lepidotteri del Delta del Niger (quarto contributo) (Arctiidae, Lithosiinae). - Lambillionea 102 (2): 135-139.

Durante, M. A. \& Panzera, S. 2002c: I Lepidotteri del Delta del Niger (Quinto contributo) (Arctiidae, Lithosiinae). - Lambillionea 102 (3): 282-286.

Durante, A. 2007: I Lepidotteri del Delta del Niger (settimo contributo). Genus Thumatha WALKER (Arctiidae, Lithosiinae). Con descrizione della nuova specie Thumatha lunaris. - Thalassia Salentina 30: 81-92.
Durante, A. 2009: Revision of the Afrotropical species of Asura Walker, 1854 (Lepidoptera: Arctiidae, Lithosiinae), with the description of a new genus. Zootaxa, 2280: 27-52.

Durante, A. 2012: New genera and problematic species in African Lithossinae (Lepidoptera, Arctiidae, Lamantriidae). - European Journal of Taxonomy 22: $1-16$.

Holloway, J. D. 2001: The moths of Borneo. 7. Family Arctiidae, subfamily Lithosiinae. - Malayan Nature Journal, Southdene Sdn. Bhd.: 208 S.

Jacobson, N. L. \& Weller, S. J. 2002: A cladistic study of the Arctiidae (Lepidoptera) by using characters of immatures and adults. - Maryland: Thomas say publications in Entomology, $97 \mathrm{~S}$.

KARISCH, T. (in prep.): Revision of the Genus Cyana in the Afrotropical region (working title).

Kirby, W. F. 1896: On a Collection of Moths from East Africa formed by Dr W. J. Ansorge, Medical Officer to the Uganda Administration. - The Annals and Magazine of Natural History (6) 18 (107): 375-396.

KiriakofF, S. G. 1954a: Hétérocères nouveaux ou peu connus du Katanga. - Revue de Zoologie et de Botanique Africaines 50 (1-2): 169-187.

KiriakofF, S. G. 1954b: Lepidoptera Heterocera. Exploration du Parc national Upemba, Miss de Witte (1946-1949), Brussels fasc. 26: 1-69.

Kiriakoff, S. G. 1958: Arctiidae (except Nolinae). Ruwenzori Expedition (1952), 1 (2): 1-39.

Kiriakoff, S. G. 1963: Lepidoptera Heterocera (partim). - Exploration du Parc National Albert 16: 73-119.

KüHne, L. 2005a: Neue Taxa und neue Synonyme afrikanischer Arctiidae (Lepidoptera). - Lambillonea 105 (3): 486-493.

KüHne, L. 2005b: Revision und Phylogenie der Gattungsgruppe Crypsotidia RothschILD, 1901, Tachosa Walker, 1869, Hypotacha Hampson, 1913, Audea WALKer, [1858] 1857 und Ulotrichopus WALLENGREN, 1860 (Lepidoptera, Noctuidae, Catocalinae) (Dissertation). - Esperiana Memoir 2: 1-222.

KüHNE, L. 2007: Beschreibung neuer Flechtenbärenarten aus Afrika nebst einigen taxonomischen Anmerkungen (Arctiidae: Lithosiinae). - Esperiana Memoir 3: 353-394

KüHNE, L. 2008 (Editor): Butterflies and moth diversity of the Kakamega Forest (Kenya). - Brandenb. Druck \& Verlagsg., $260 \mathrm{~S}$.

Lafontaine, J. D. \& Fibiger, M. 2006: Revised higher classificatio of the Noctuoidea (Lepioptera). - Canadian Entomology 138: 610-635.

GÜSSFELdT, P. 1879: Die Loango-Expedition. - In: Paul Güssfeldt, Julius Falkenstein, Eduard Pechuël-Loesche (1879-1882): Die Loango-Expedition. Ausgesandt von der Deutschen Gesellschaft zur Erforschung Aequatorial-Africas 1873-1876. Ein Reisewerk in drei Abtheilungen. - Leipzig: Paul Frohberg. Erste Abtheilung, 1879: $161 \mathrm{~S}$. 


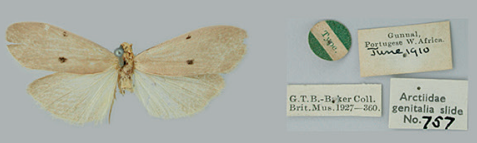

1 ơ diplisticta $(\mathrm{HT})$

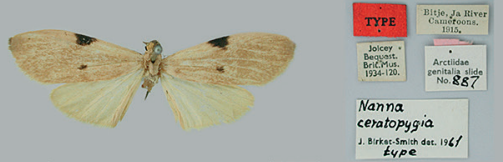

4 ô ceratopygia (HT)

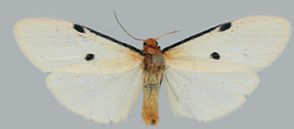

7 ổ broetheri (HT)

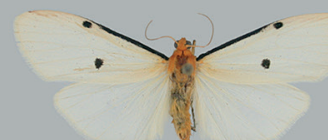

8 qbroetheri (PT)

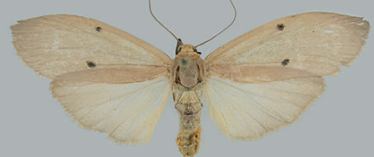

2 \& diplisticta

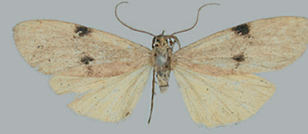

5 ô ceratopygia

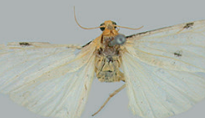

9 ô colonoides $(\mathrm{HT})$

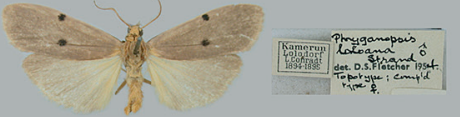

3 o loloana

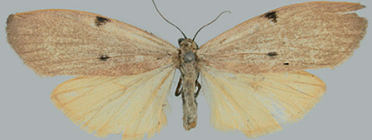

6 q ceratopygia

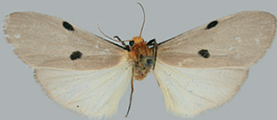

11 ô colonoides

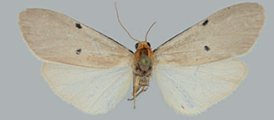

12 ô colonoides

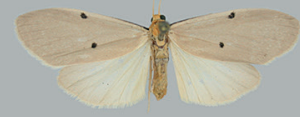

13 q colonoides

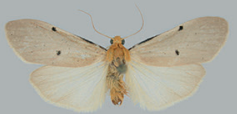

14 ồ naumanni $(\mathrm{HT})$

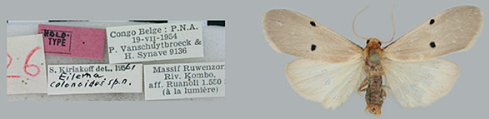

10 ô colonoides

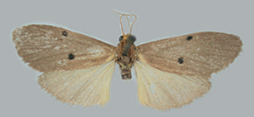

16 hoppei $(\mathrm{HT})$

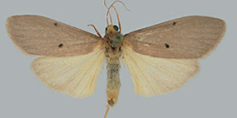

17 กิ hoppei (PT)

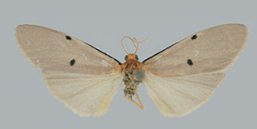

18 ๙ิ hoppei (PT)

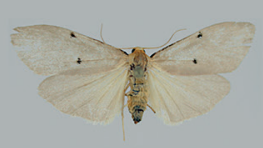

19 q hoppei

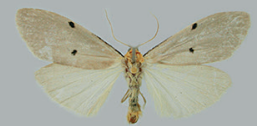

15 ô naumanni (PT)

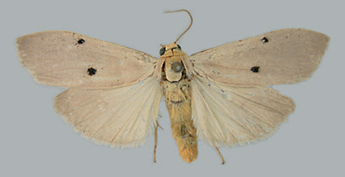

21 ô griseata (PT)

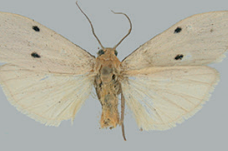

25 ơ griseata

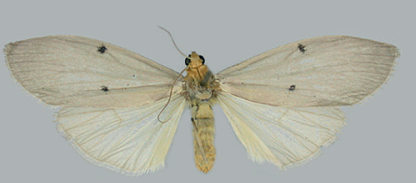

29 o distyi

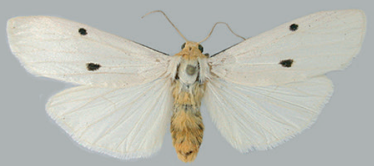

32 ô collinsii $(\mathrm{PT})$

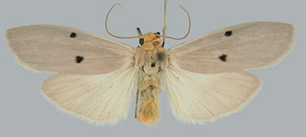

22 ô $_{\text {griseata }}$

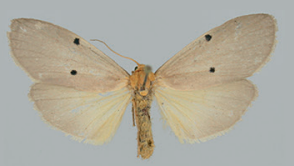

26 griseata

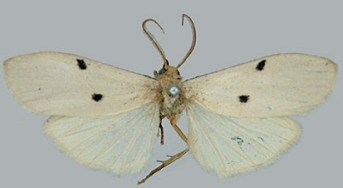

30 ô melanosticta $(\mathrm{HT})$

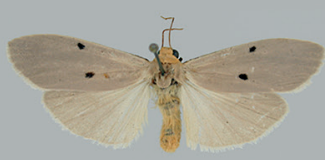

23 ô griseata $(\mathrm{PT})$

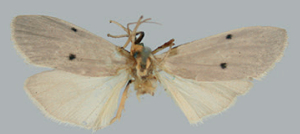

27 ô distyi (HT)

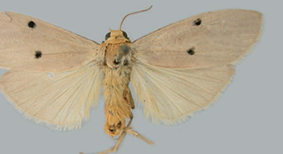

$20 \partial^{\lambda}$ griseata $(\mathrm{HT})$

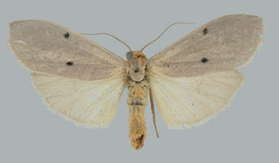

24 ò griseata

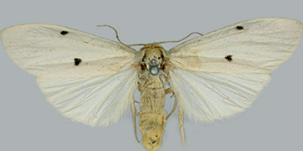

28 ơ distyi (PT)

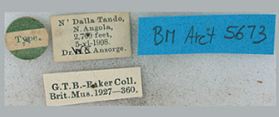

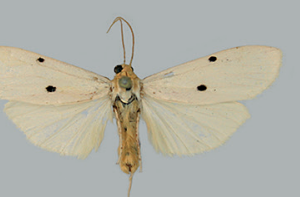

31 ô collinsii (PT)

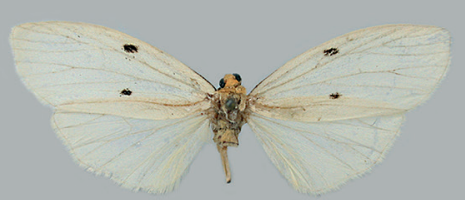

33 q collinsii

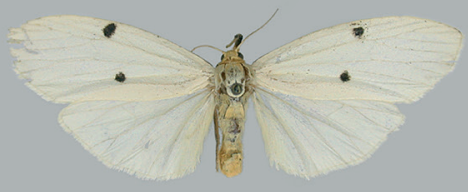

34 o collinsii

Tafel 1 


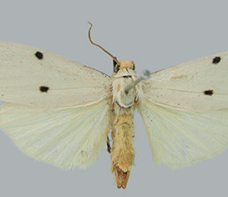

35 ô pia $(\mathrm{HT})$

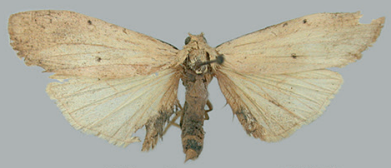

38 ô magna $(\mathrm{HT})$

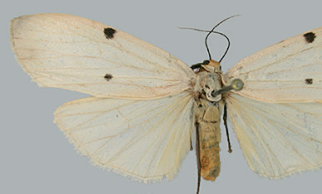

40 o eningae

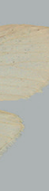

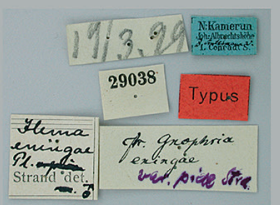
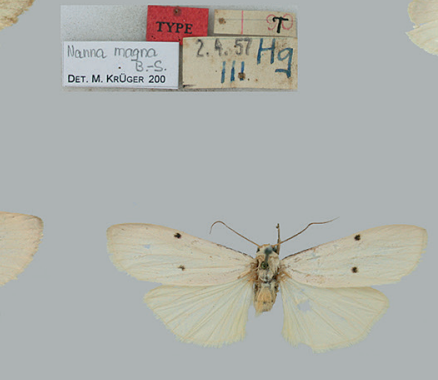

41 ô eningae

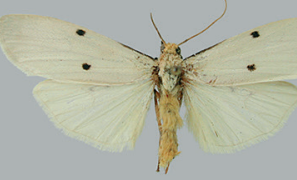

36 ô pia

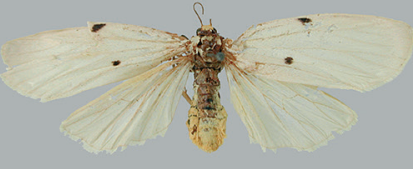

39 ㅇ eningae $(\mathrm{HT})$

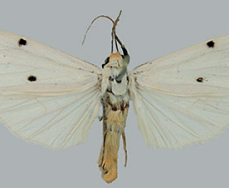

42 ô eningae

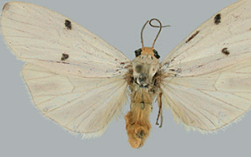

37 ô pia

reo

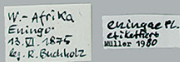

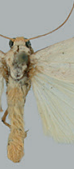

43 ô eningae

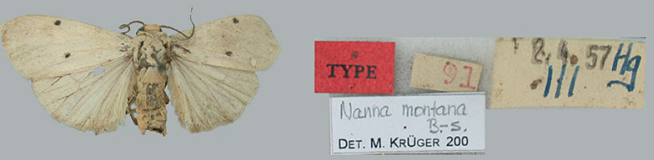

44 ô montana $(\mathrm{HT})$

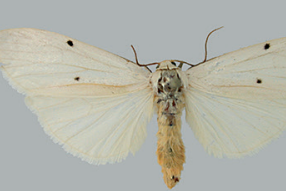

45 ỏ albifrons (PT)

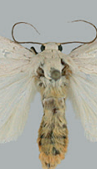

46 ôlbifrons (PT)
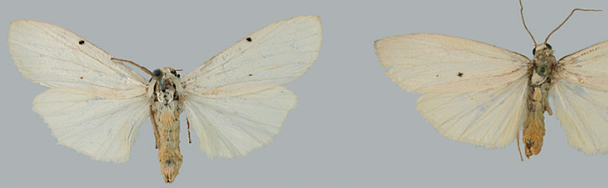

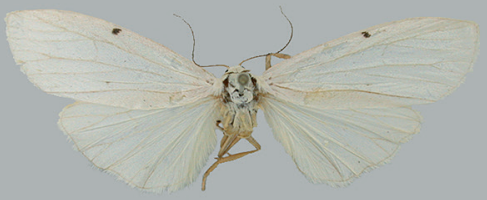

49 i albifrons (PT)

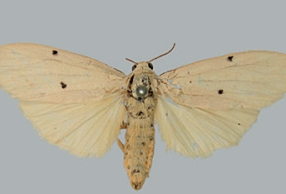

52 ô subflava $(\mathrm{PT})$

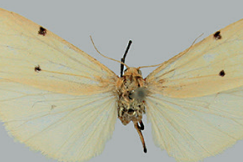

56 甲 tanzaniae (HT)

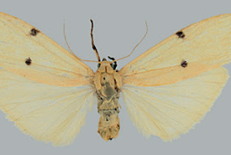

60 ổ luteolata (PT)

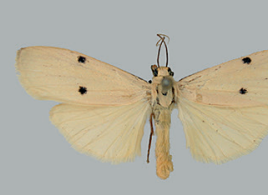

53 ô subflava (HT)

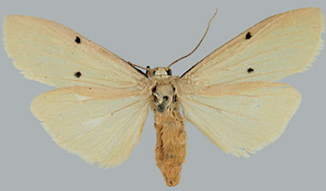

57 ồ tanzaniae (PT)

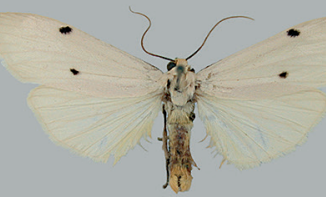

61 kamerunica (PT)
48 ô albifrons (PT)

47 ¿̂ albifrons (PT)

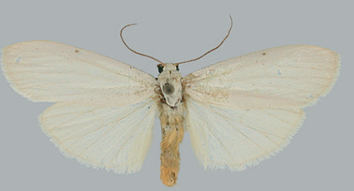

51 ôalbifrons (PT)

50 i albifrons (PT)

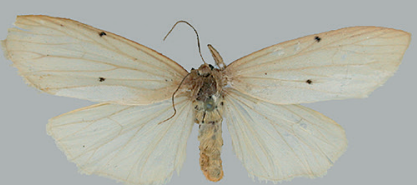

55 subflava $(\mathrm{PT})$
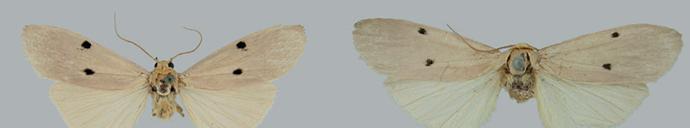

59 ô griseoides (PT)

58 ô griseoides (HT)

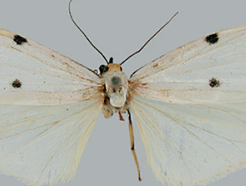

63 of falcata (PT) 
PLöTz, C. 1879: Verzeichniss der vom verstorbenen Prof. Dr. R. Buchholz in West-Afrika - beim Meerbusen von Guinea - gesammelten Hesperien. - Stettiner Entomologische Zeitung 40: 353-364.

Plötz, C. 1880a: Verzeichniss der vom Professor Dr. R. Buchholz in West-Afrika gesammelten Schmetterlinge. - Stettiner Entomologische Zeitung, 41: 76-88, 298-307.

Plötz, C. 1880b: Verzeichniss der vom Professor Dr. R. Buchholz in West-Afrika - vom 5. Gr. nördl. bis 3. Gr. südl. Breite, auf dem Cameroons-Gebirge in ungefährer höhe von 4000 Fuss und auf der Insel Fernando Po, vom August 1872 bis November 1875 gesammelten Schmetterlinge. - Stettiner Entomologische Zeitung 41: 189-206.
Plötz, C. 1880c: Berichtigungen und Bemerkungen zum Verzeichnis der vom Professor Buchholz in West-Afrika gesammelten Schmetterlinge. - Stettiner Entomologische Zeitung 41 (10-12): 477-478.

Roesler, R. U. 1990: Die Cyana-Spezies von Afrika. Teil 1: Zwei neue Arten aus den Beständen des Naturhistorischen Museums in Budapest. - Entomofauna 11 (10): 161-175.

Strand, A. 1912: Zur Kenntnis äthiopischer Lithosiinae. - Archiv für Naturgeschichte 78 (7): 171-195.

Seitz, A. 1926: 4. Familie: Arctiidae, Bärenspinner: 61-88. - In: Seitz, A. (1913-1940): Die GrossSchmetterlinge der Erde, 14. Die Afrikanischen Spinner und Schwärmer. - Stuttgart: Alfred Kernen, iii +599 S., 80 Tafeln. 


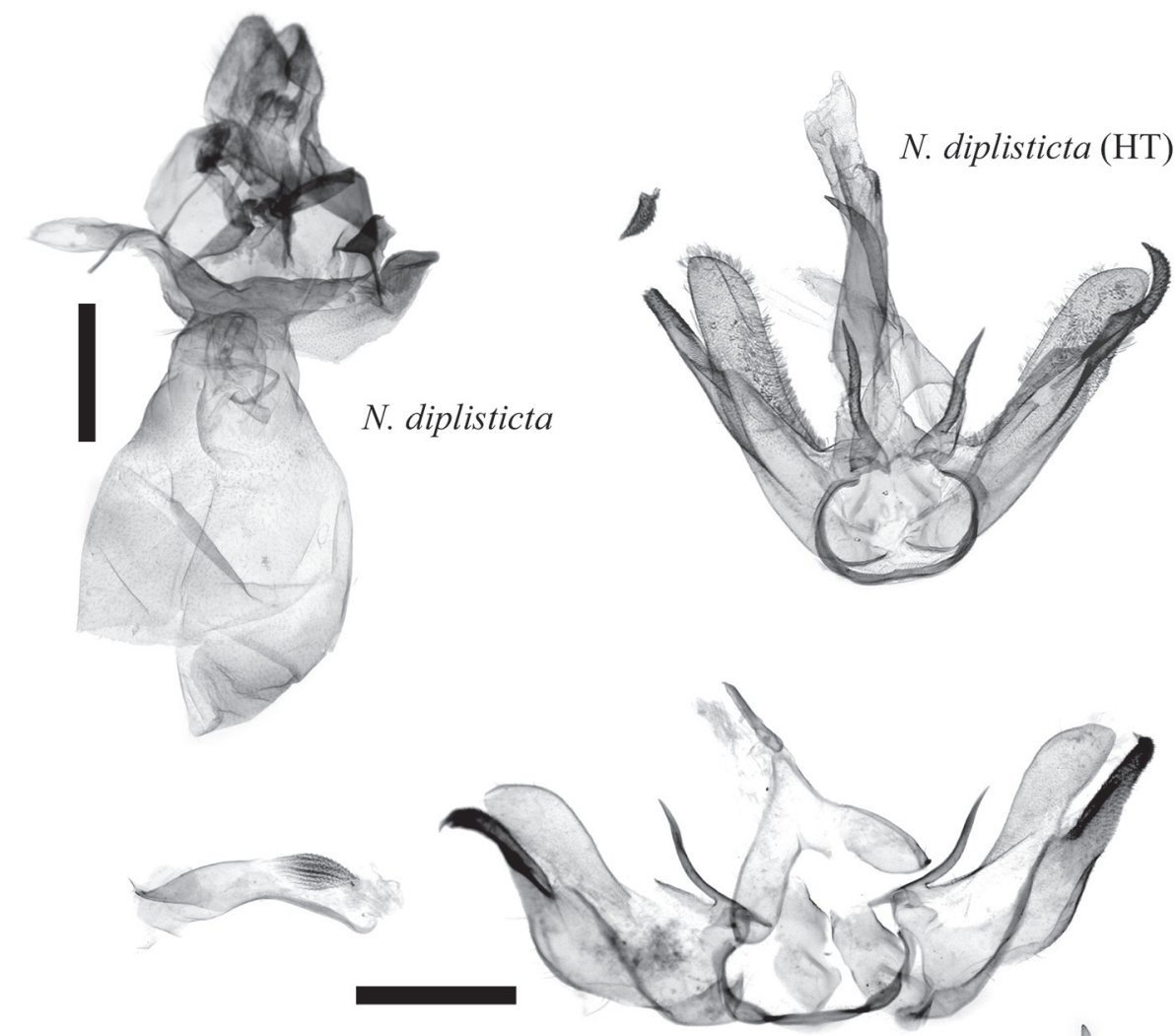

N. loloana ("Syntypus")
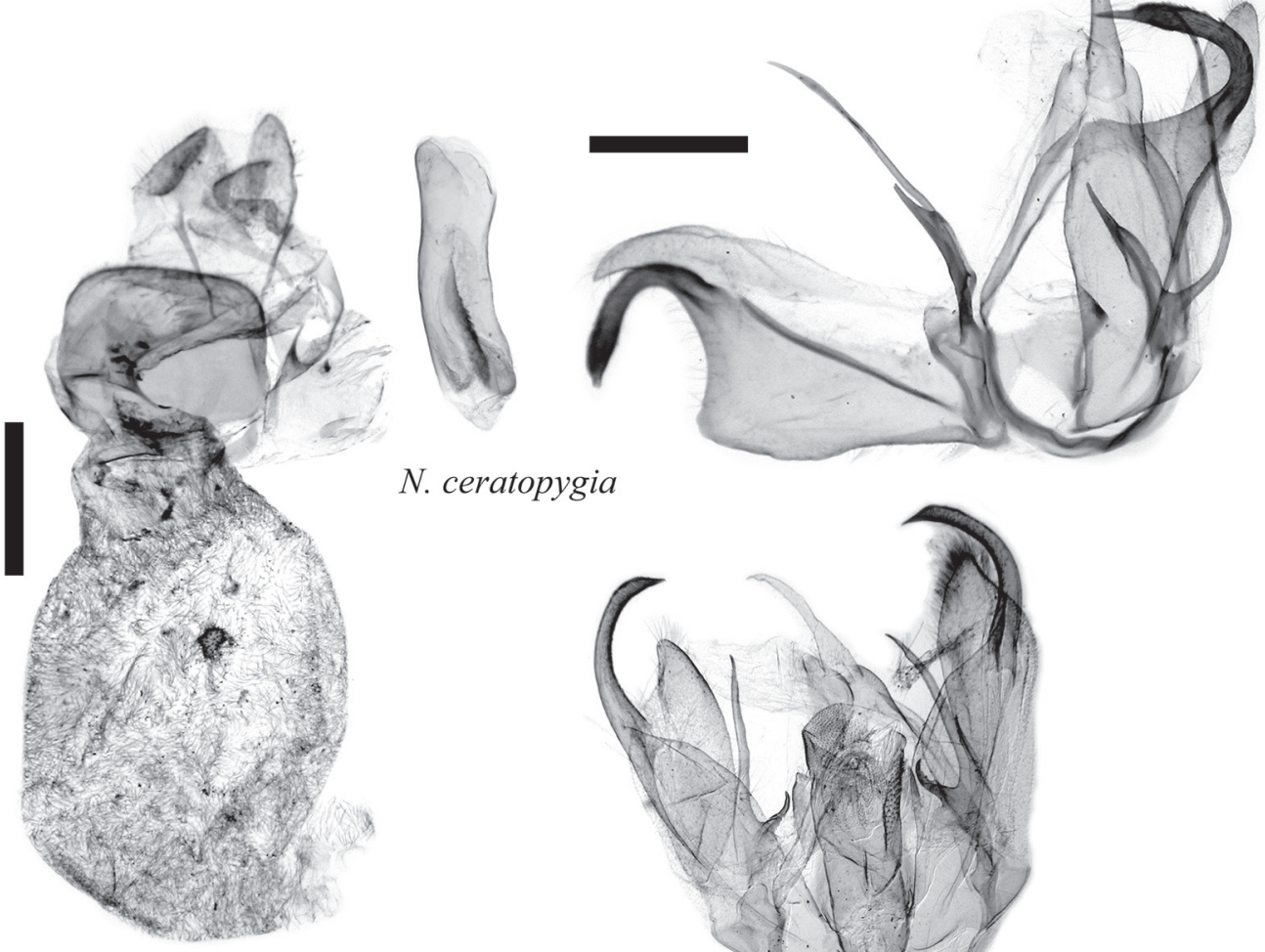

N. ceratopygia

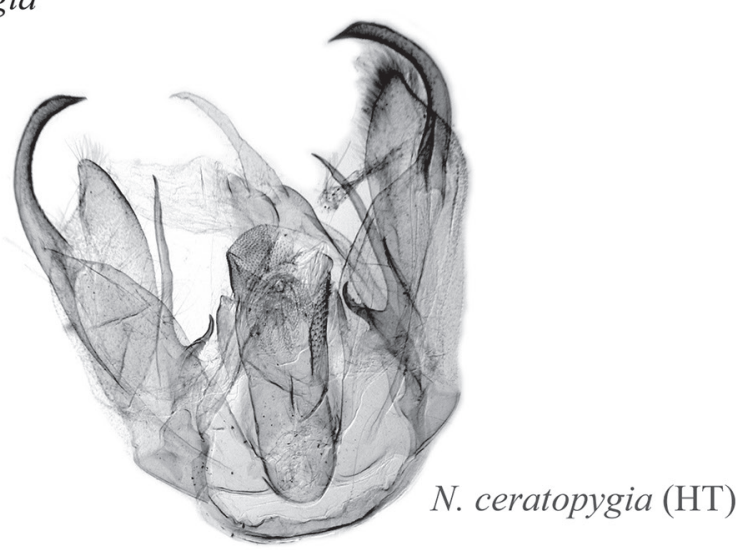

Tafel 3 

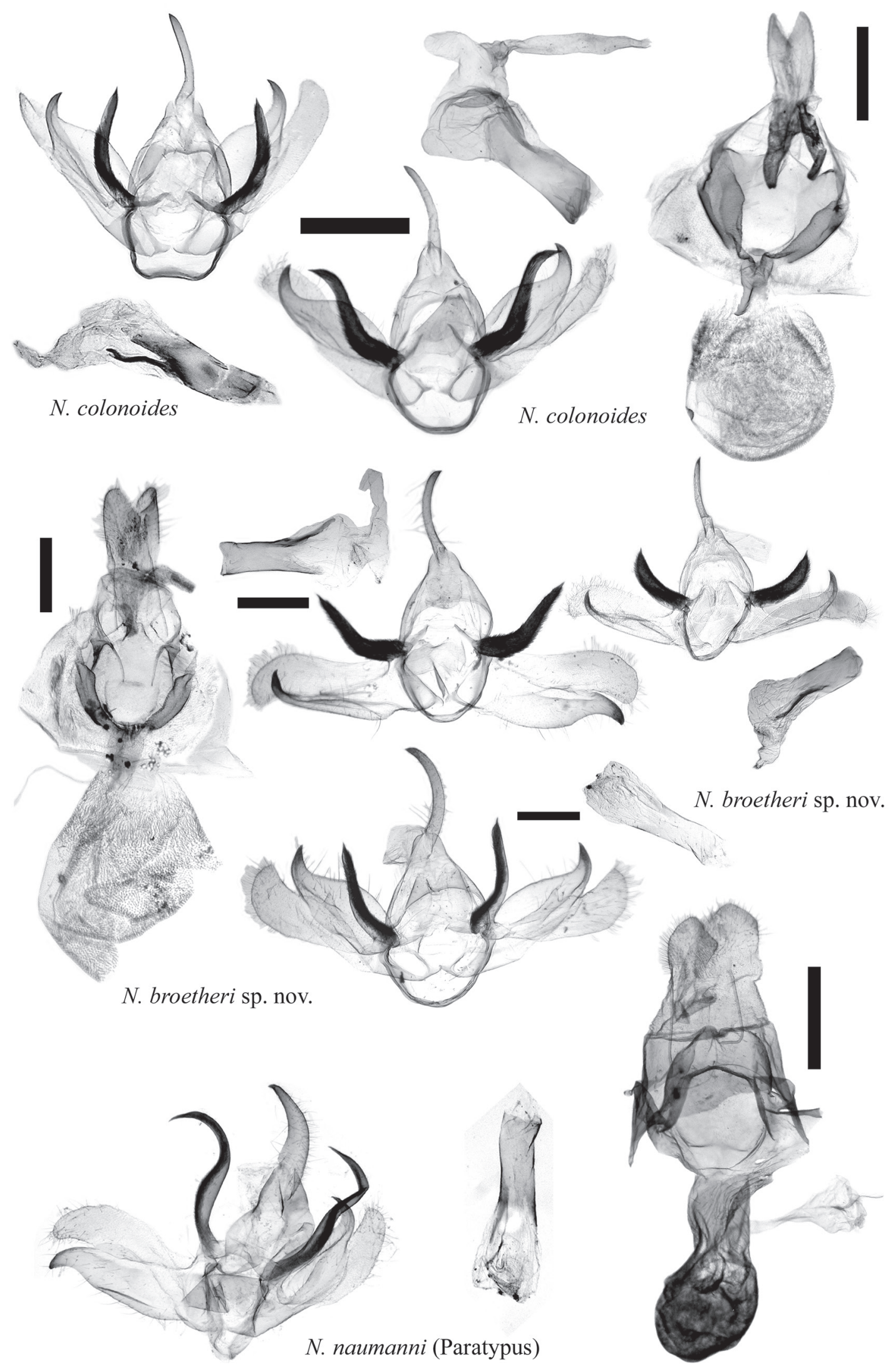

Tafel 4 


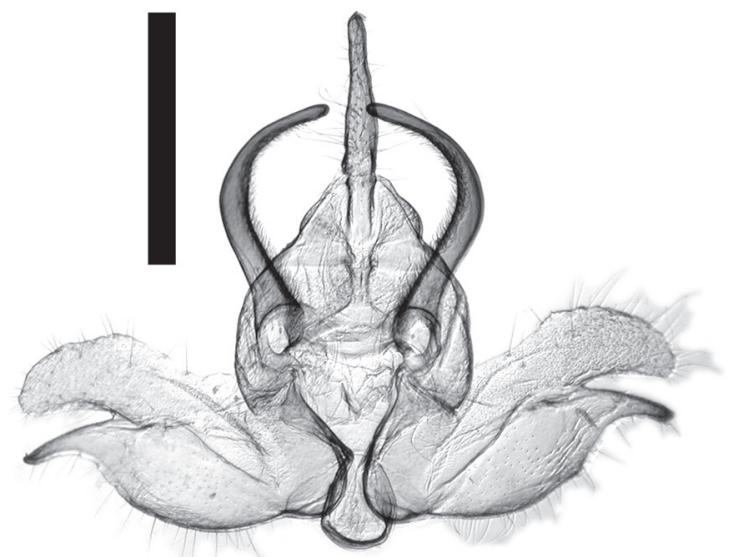

N. hoppei sp. nov.
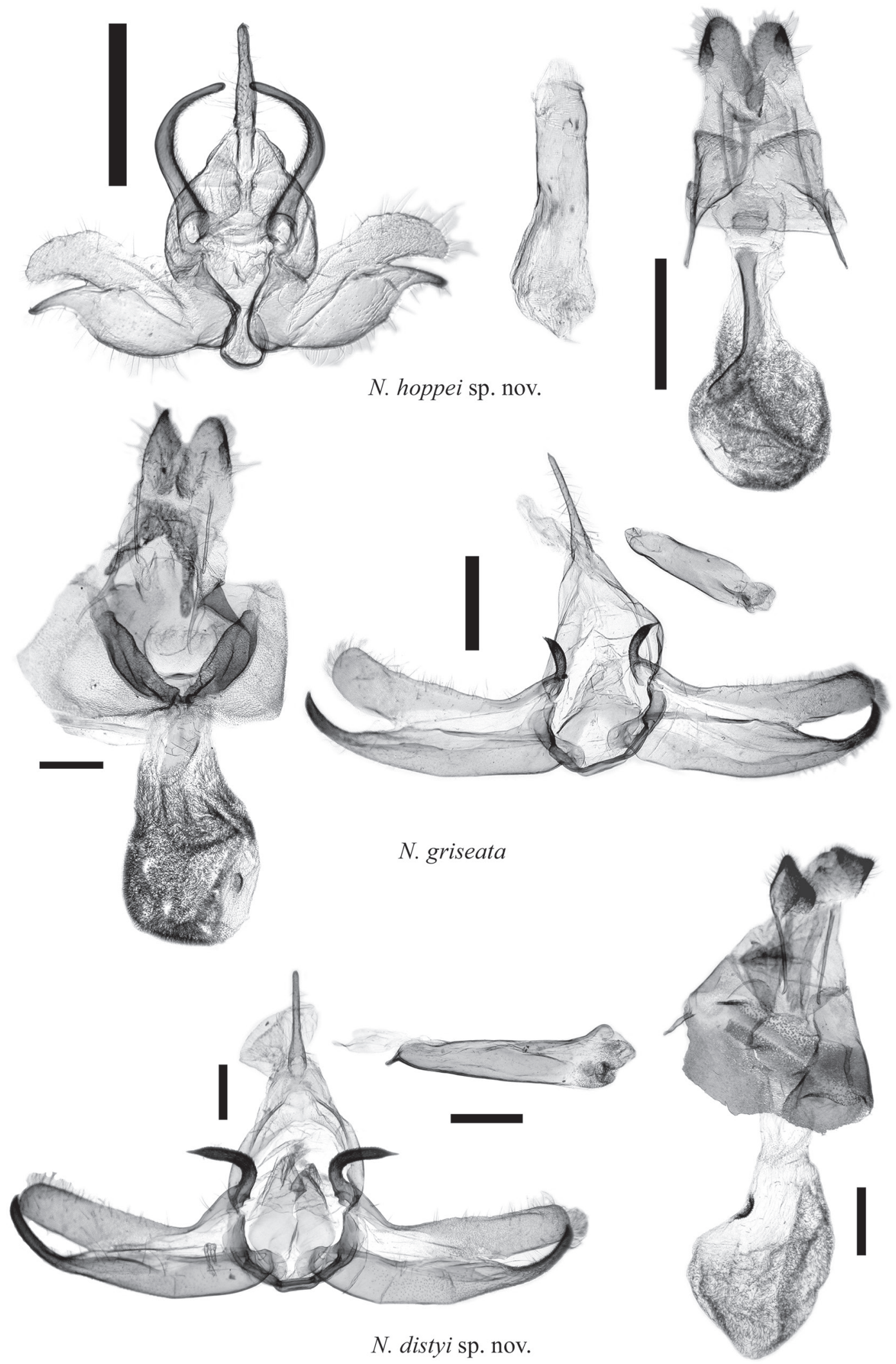

Tafel 5 

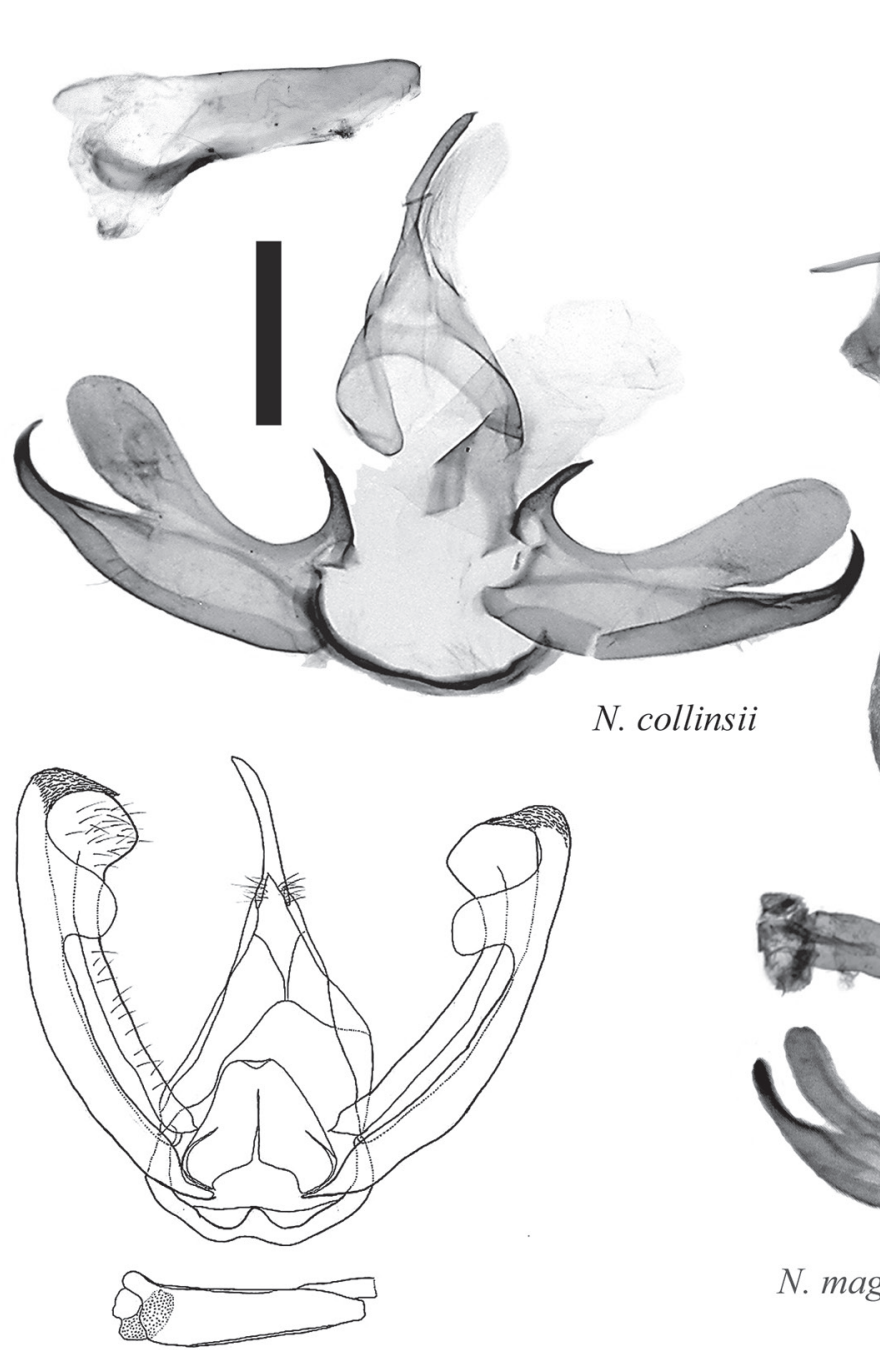

N. magna (Typus)

N. melanosticta (HT, Zeichnung: M. Krüger)

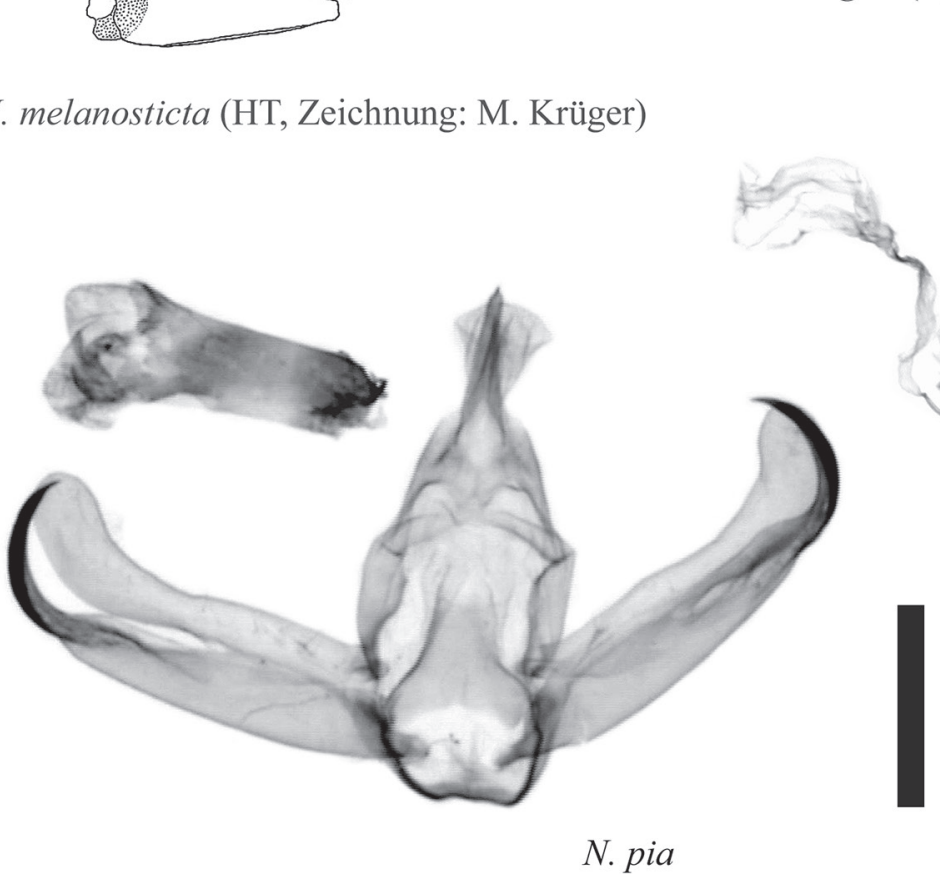

N. pia
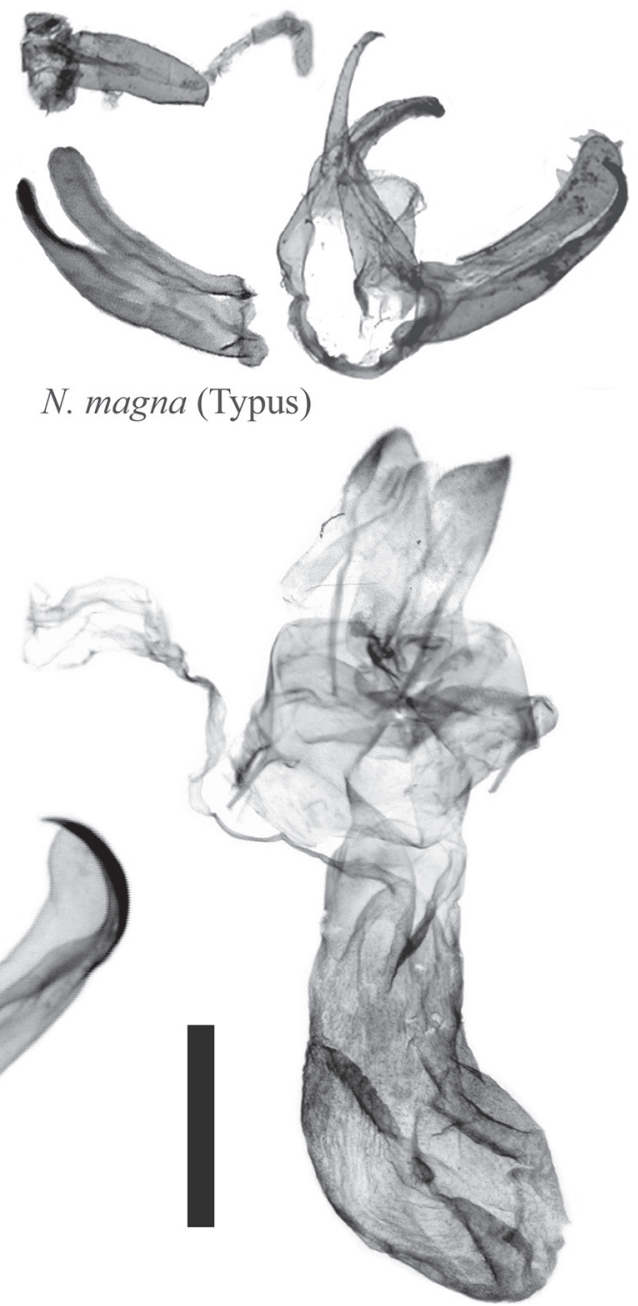

Tafel 6 

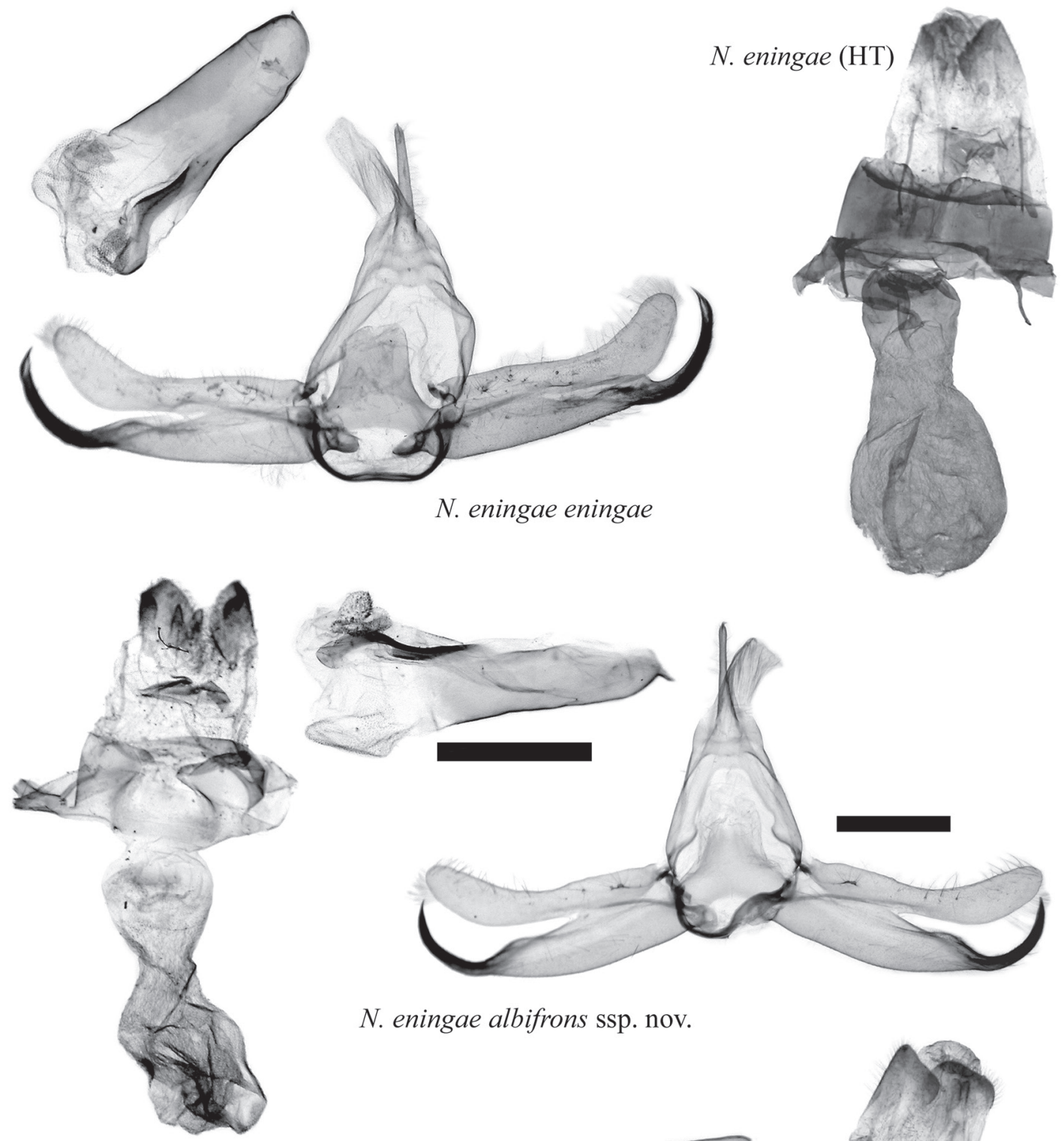

$N$. eningae albifrons ssp. nov.

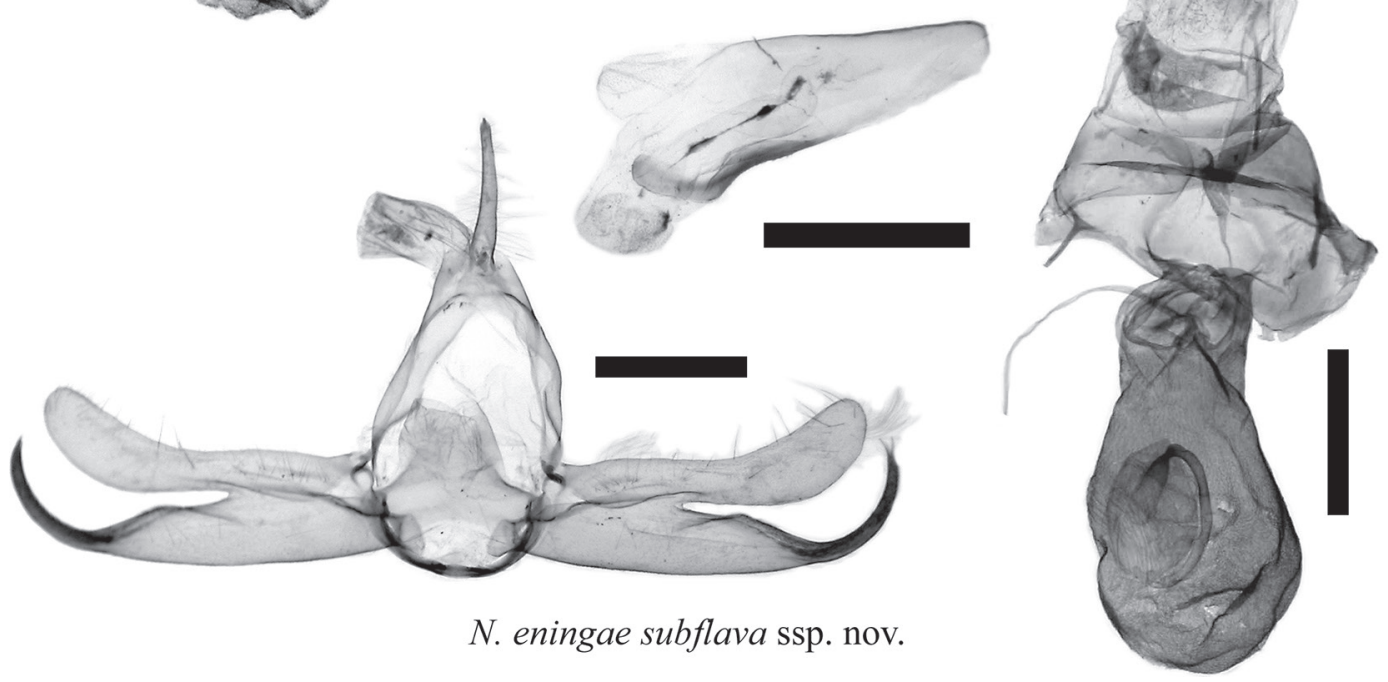

Tafel 7 

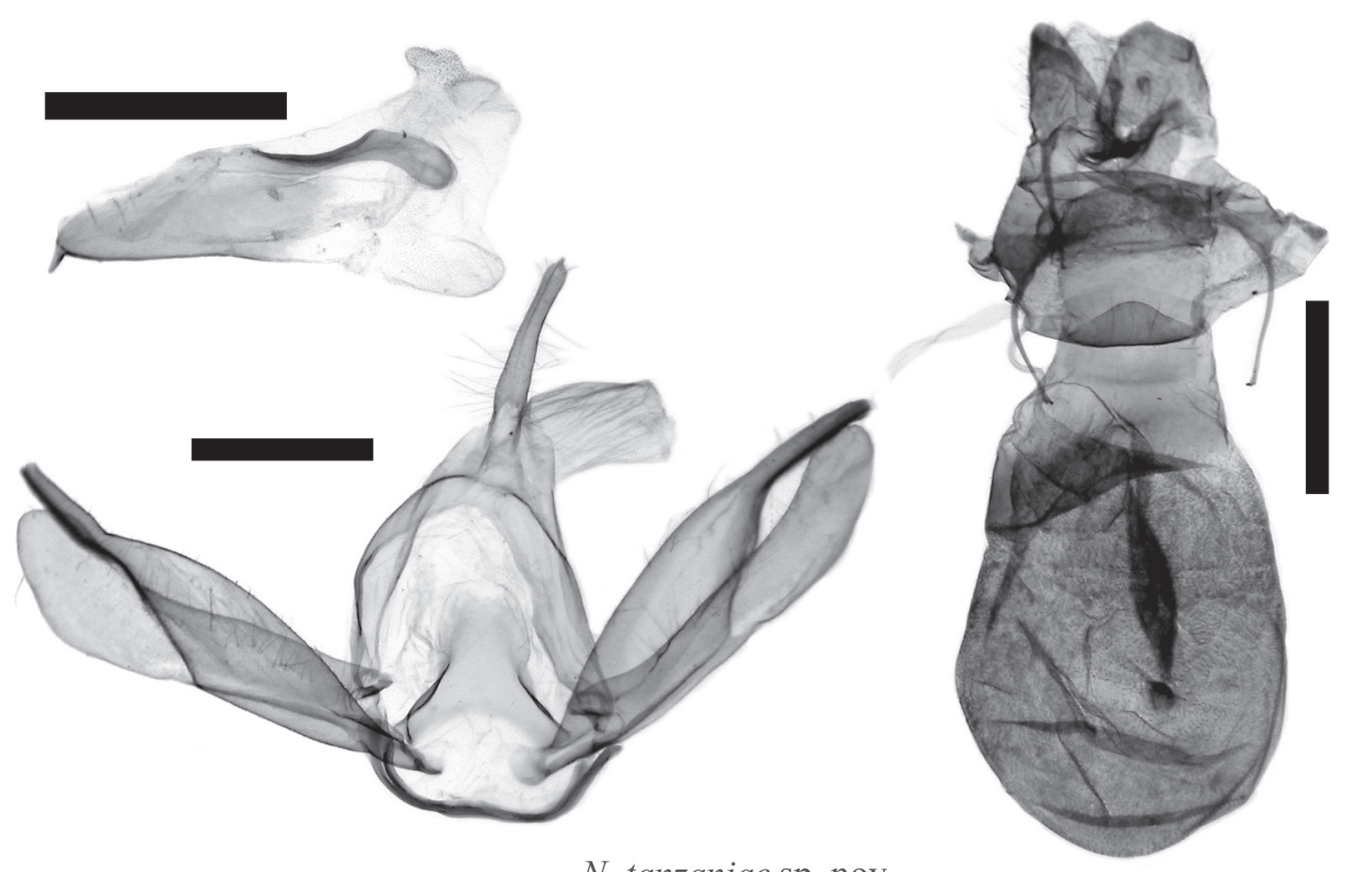

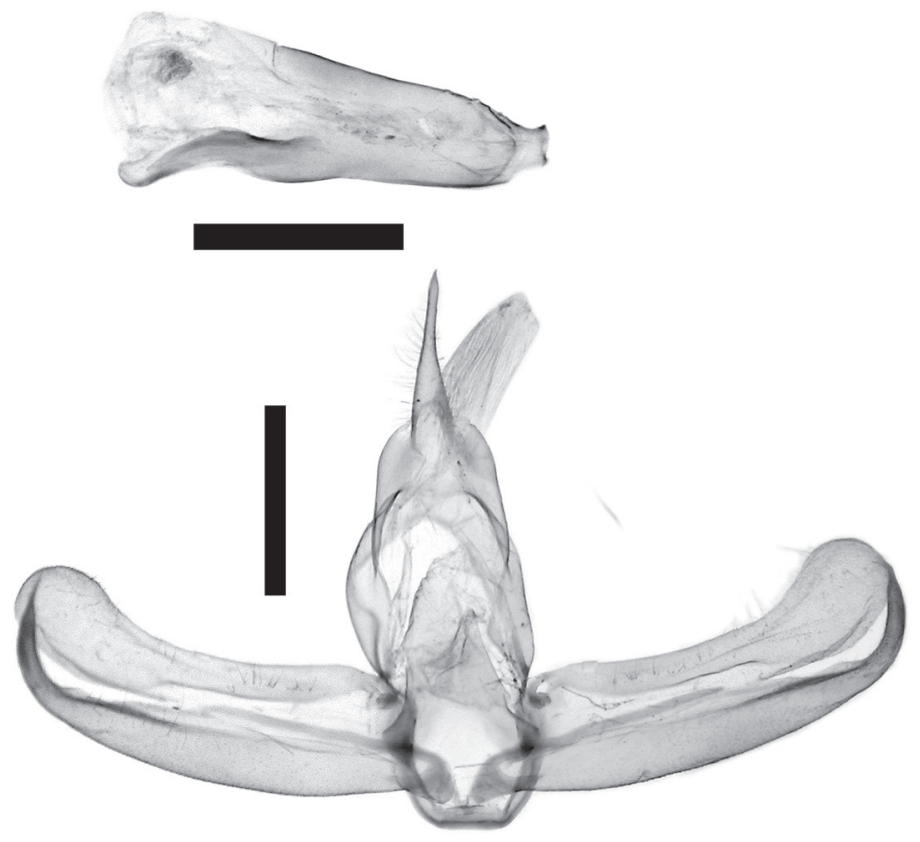

N. griseoides sp. nov.

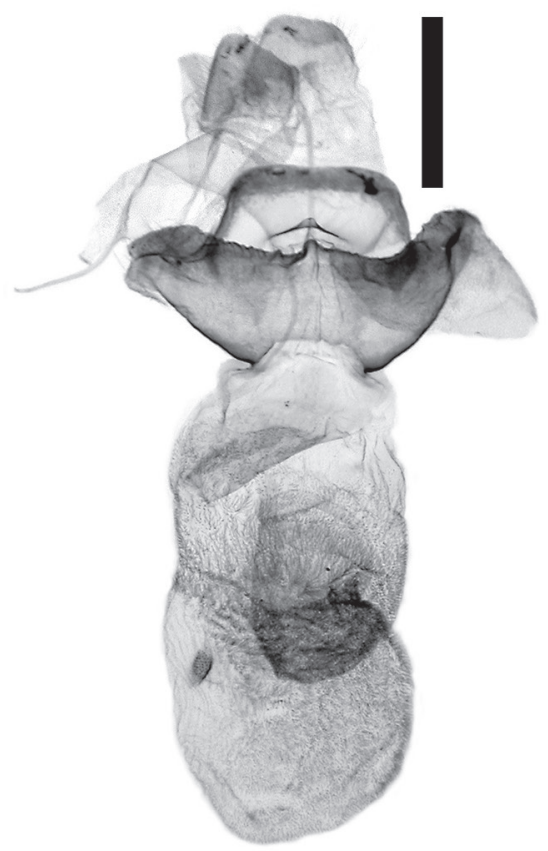

N. luteolata sp. nov.

Tafel 8 

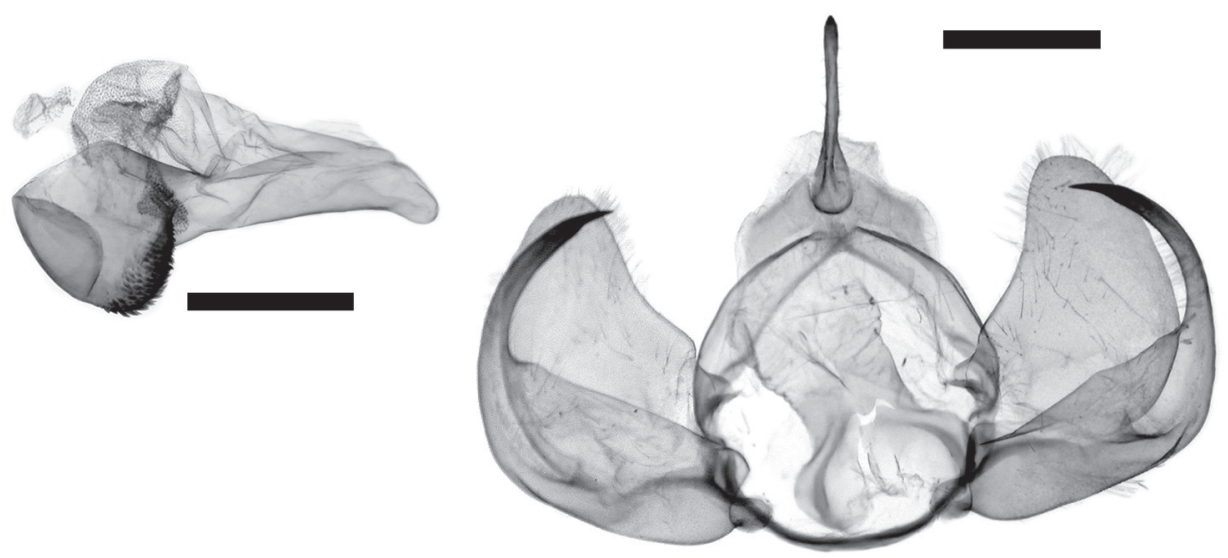

N. kamerunica $(\mathrm{HT})$

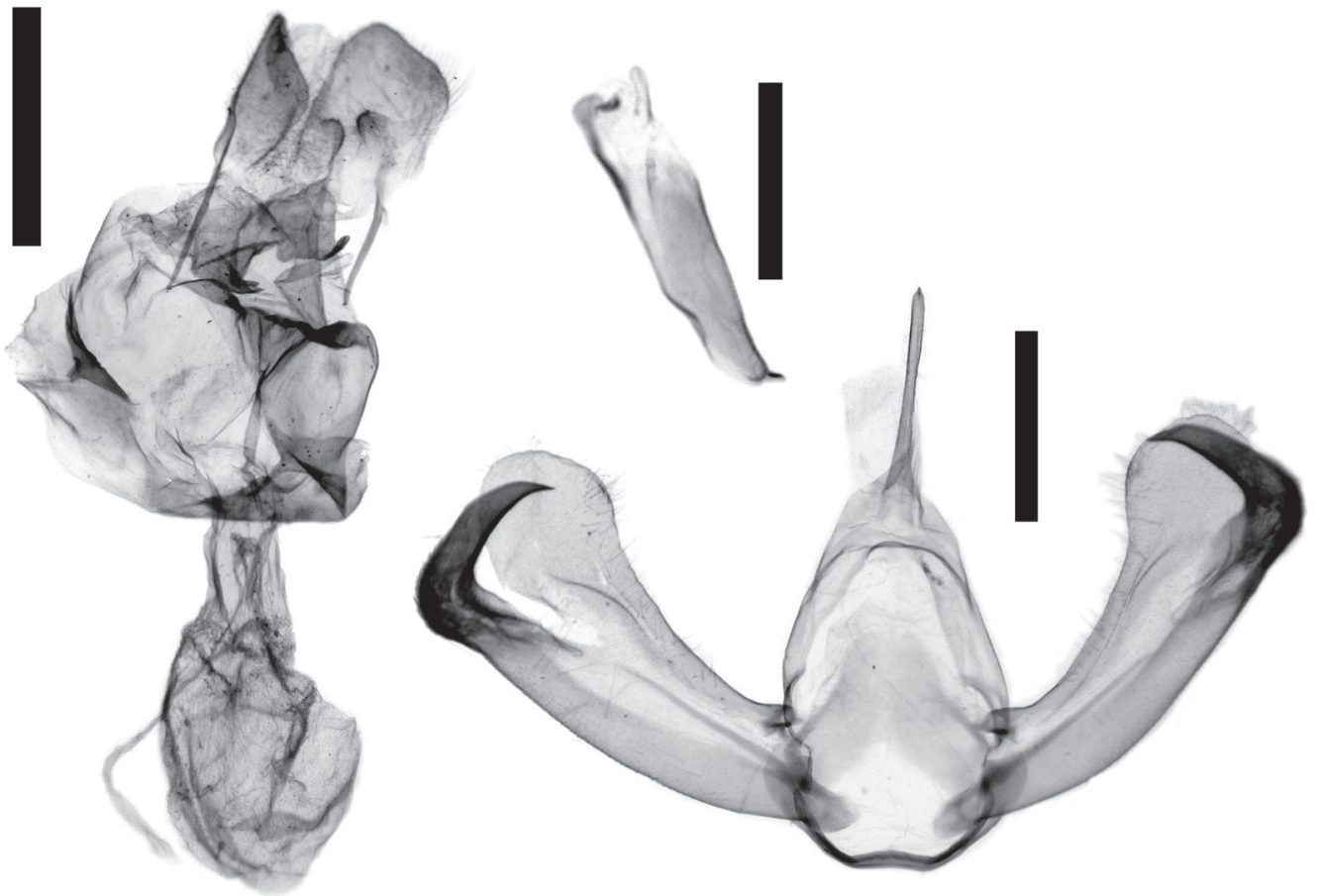

N. falcata sp. nov.

Tafel 9 